\title{
Image Segmentation Using CUDA Accelerated Non-local Means Denoising and Bias Correction Embedded Fuzzy C-Means (BCEFCM)
}

\author{
Chaolu Feng ${ }^{\mathrm{a}, \mathrm{b}, *}$, Dazhe Zhao ${ }^{\mathrm{a}, \mathrm{b}}$, Min Huang ${ }^{\mathrm{a}, \mathrm{c}}$ \\ ${ }^{a}$ College of Information Science and Engineering, Northeastern University, Shenyang, Liaoning, 110819, China \\ ${ }^{b}$ Key Laboratory of Medical Image Computing of Ministry of Education, Northeastern University, Shenyang, Liaoning, \\ 110819, China \\ ${ }^{c}$ State Key Laboratory of Synthetical Automation for Process Industries, Northeastern University, Shenyang, Liaoning, \\ 110819, China
}

\begin{abstract}
Due to intensity overlaps between interested objects caused by noise and intensity inhomogeneity, image segmentation is still an open problem. In this paper, we propose a framework to segment images in the well-known image model in which intensities of the observed image are viewed as a product of the true image and the bias field. In the proposed framework, a CUDA accelerated non-local means denoising method is first used to remove noise from the image. Then, a bias correction embedded fuzzy c-means (BCEFCM) method is proposed to segment the image and correct the bias field simultaneously. To ensure the slowly and smoothly varying property of the bias field, we convolve it with a normalized kernel as soon as it is updated in each iteration. The proposed framework has been extensively tested on both selected synthetic and real images and public BrainWeb and IBSR datasets. Experimental results and comparison analysis demonstrate that the proposed framework is not only able to deal with noise and correct the bias field but it is also faster and more accurate than state-of-the-art methods.
\end{abstract}

Keywords: image segmentation, bias correction, fuzzy c-means, non-local means denoising, CUDA acceleration

\section{1. introduction}

Image segmentation is fundamental and important for computer vision, pattern recognition, and medical

\footnotetext{
${ }^{*}$ Corresponding author

Email address: fengchl@ise.neu.edu.cn (Chaolu Feng)
} 
NMF) [5, 6, 7], model-based methods [8, 9], atlas-based methods [10, 11], machine learning methods (for example, multi-modal/cross-modal learning [12, 13] and multi-view learning [14, 15, 16]), active contour and level set methods [17, 18, 19], and clustering methods [20, 21]. But due to overlaps between intensity distribution of interested objects caused by noise and intensity inhomogeneity (also known as bias field) as shown in Fig. 1, image segmentation is still an open problem [22].
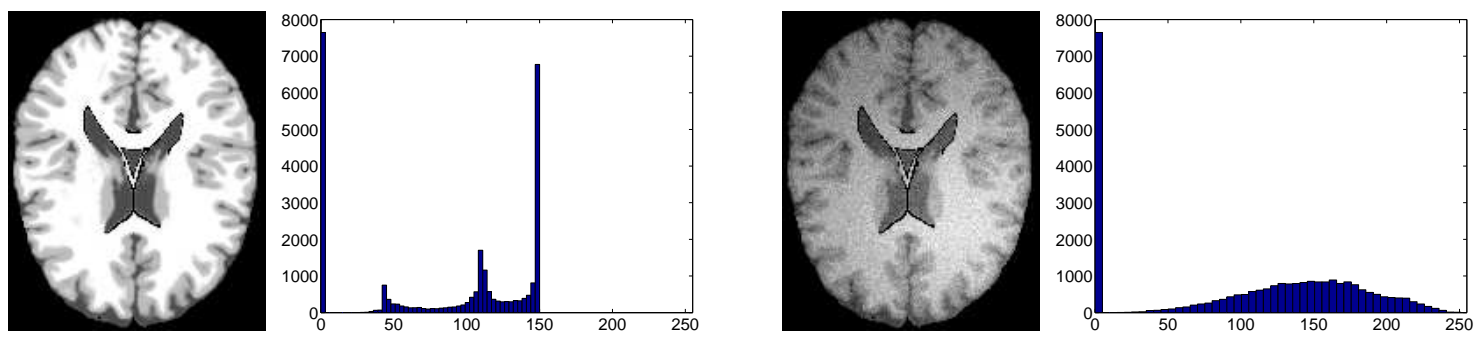

Figure 1: Appearance and intensity histograms of a brain image corrupted by both noise and intensity inhomogeneity (right) or neither (left).

As one of the well known clustering methods, fuzzy c-means (FCM) has been widely studied in the literature for its application to image segmentation [23]. In FCM, each pixel is assigned to all desired clusters with memberships graded as fuzzy values between 0 and 1 [24, 25]. Note that this is different from hard c-means in which each pixel is assigned exactly to only one cluster with the membership graded as either 0 or 1 [26]. But FCM and hard c-means are both sensitive to noise and lacks the ability to deal with intensity inhomogeneity because they classify pixels independently instead of considering the correlation among neighbouring pixels [27]. To overcome this drawback, Ahmed et al. proposed a bias corrected fuzzy c-means (BCFCM) method in [28]. In BCFCM, the objective function of the standard FCM is modified by incorporating a spatial constraint to compensate for the intensity inhomogeneity with neighbouring intensities and to bias the solution toward piecewise-homogeneous labellings [28]. Chen and Zhang modified BCFCM by replacing each pixel in the spatial constraint with the mean or median intensity of neighbours around the pixel and computed a corresponding mean- or median-filtered image in advance to improve the time performance of BCFCM [29]. They then replaced the Euclidean distance with a Gaussian kernelinduced distance and proposed kernel versions of FCM with spatial constraints to enhance the robustness of BCFCM to noise and outliers [29]. However, final clustering results of methods proposed by Ahmed et al. and Chen and Zhang are both heavily affected by the parameter used to adjust influence of the spatial constraint. Therefore, Yang and Tsai proposed a generalized type of BCFCM in [30] where the parameter is estimated automatically under a learning scheme. However, all the above mentioned improvements of BCFCM are without pay any attention to correct the bias field from the image. Hence, Li et al. proposed a modified FCM model which is named as multiplicative intrinsic component optimization (MICO) model in 31] to segment images with intensity inhomogeneity and correct the bias field. In MICO, the slowly and smoothly varying property of the bias field is ensured by a linear combination of a given set of smooth basis 
functions.

In this paper, we propose a framework to segment images with both noise and intensity inhomogeneity. In the framework, intensities of a given image is viewed as a product of the true signal generated by the underlying anatomy and the bias field. Noise are considered as an additive component in the image model, which are first removed by CUDA accelerated non-local means denoising (NLMD) method. A bias correction embedded FCM (BCEFCM) method is then proposed to segment the noise removed image with intensity inhomogeneity and correct the bias field. Finally, a coefficient for each cluster is introduced to control size of the cluster.

The rest of this paper is organized as follows. We first briefly review the standard FCM and some state-of-the-art FCM based methods in section 2. In section 3, we first give the non-local means denoising algorithm and then accelerate it with CUDA and apply it to remove noise from noisy images. We then propose an improved FCM method named BCEFCM to segment the noise removed images and estimate the bias field and finally introduce a weighting coefficient for each cluster to control the amount of pixels that will be considered as the cluster. Experimental results of NLMD+BCEFCM on several synthetic and real images and comparison of results obtained by the proposed framework with state-of-the-art methods are given in section 4. To analyse the performance of NLMD+BCEFCM quantitatively, we finally applied it to BrainWeb and IBSR datasets in section 4 and compare the results with state-of-the-art methods in false positive ratio, false negative ratio, and dice similarity coefficient. We discuss the robustness of BCEFCM to initialization and impacts of weighting coefficients and other control parameters on segmentation and bias correction results in section 5. This paper is finally summarized in section 6.

\section{Related Work}

Given an observed image $I$ defined on a continuous domain $\Omega \subset R^{2}$ with intensity inhomogeneity, its intensities can be viewed as a product of the true image $J$ and the bias field $b$, i.e,

$$
I(\mathbf{x})=b(\mathbf{x}) J(\mathbf{x})+n(\mathbf{x})
$$

\footnotetext{
where $\mathbf{x} \in \Omega$ and $n$ is zero-mean additive noise. The true image $J$ characterizes an intrinsic physical property of objects being imaged, which ideally takes a specific intensity $c_{i}$ for the $i$-th type of objects and is therefore assumed to be piecewise constant. That is to say, the true image $J$ approximately takes $N$ distinct constant values $c_{1}, c_{2}, \ldots$, and $c_{N}$ in disjoint regions $\Omega_{1}, \Omega_{2}, \ldots$, and $\Omega_{N}$, respectively. The bias field $b$ is assumed to be slowly and smoothly varying as well known in the literature. The problem of image segmentation and bias correction is therefore considered as finding the specific intensities $c_{1}, c_{2}, \ldots$, and $c_{N}$ and estimating the bias field $b$.
} 


\subsection{Fuzzy C-Means}

As mentioned above, true intensity of the observed image $I$ at location $\mathbf{x}$ can be represented by $J(x)=$ $\sum_{i=1}^{N} c_{i} u_{i}(\mathbf{x})$ where $u_{i}(\mathbf{x})$ is the membership function that indicates whether pixel $\mathbf{x}$ belongs to the $i$-th object or not. In the ideal case, the membership function $u_{i}(\mathbf{x})$ is a boolean function. That is to say, $u_{i}(\mathbf{x})=1$ if $\mathbf{x}$ belongs to the $i$-th object and $u_{i}(\mathbf{x})=0$ otherwise. In the real case, fuzzy clustering are able to classify each pixel to more than one cluster with a set of membership levels which indicates the strength of association between the pixel and its cluster centroid. As one of the most widely used fuzzy clustering algorithms, the objective function of the standard FCM for partitioning the observed image $I$ into $N$ clusters can be written into the following continuous form:

$$
F_{f c m}=\int_{\Omega} \sum_{i=1}^{N}\left\|I(\mathbf{x})-c_{i}\right\|^{2} u_{i}^{q}(\mathbf{x}) d \mathbf{x}
$$

where $q$ is any real number that is not less than 1 , and $\|*\|$ is one norm expression of the similarity (Euclidean distance in general) between measured intensity $I(\mathbf{x})$ and the cluster centroid $c_{i}$. This objective function is minimized when high membership values are assigned to pixels, intensities of which are close to the centroid, and low membership values are assigned when the pixels are far from the centroids under the condition

$$
\sum_{i=1}^{N} u_{i}(\mathbf{x})=1
$$

where $u_{i}(\mathbf{x}) \in[0,1]$.

The minimization of the objective function $F_{f c m}$ is achieved by an iterative optimization procedure. For other variables fixed, we minimize the energy $F_{f c m}$ with respect to $c_{i}$ by resolving $\frac{\partial F_{f c m}}{\partial c_{i}}=0$ and obtain the minimizer $\tilde{c}_{i}$ of the objective function $F_{f c m}$, given by

$$
\tilde{c}_{i}=\frac{\int_{\Omega} I(\mathbf{x}) u_{i}^{q}(\mathbf{x}) d \mathbf{x}}{\int_{\Omega} u_{i}^{q}(\mathbf{x}) d \mathbf{x}}, \quad i=1,2, \ldots, N
$$

For other variables fixed, the energy $F_{f c m}$ can be minimized with respect to $u_{i}(\mathbf{x})$ subject to the constraint that $\sum_{i=1}^{N} u_{i}(\mathbf{x})=1$. For the case $q>1$, the energy functional $F_{f c m}$ is minimized by $\tilde{u}_{i}(\mathbf{x})$, given by

$$
\tilde{u}_{i}(\mathbf{x})=\frac{\left(\left\|I(\mathbf{x})-c_{i}\right\|^{2}\right)^{\frac{1}{1-q}}}{\sum_{j=1}^{N}\left(\left\|I(\mathbf{x})-c_{j}\right\|^{2}\right)^{\frac{1}{1-q}}}, \quad i=1,2, \ldots, N
$$

For $q=1$, it can be shown that the minimizer $\tilde{u}_{i}(\mathbf{x})$ is given by

$$
\tilde{u}_{i}(\mathbf{x})=\left\{\begin{array}{ll}
1, & i=i_{\text {min_- } f c m(\mathbf{x})} \\
0, & i \neq i_{\text {min_f }_{\text {- }}(\mathbf{x})}
\end{array} \quad i=1,2, \ldots, N\right.
$$


where

$$
i_{m i n_{-} f c m}(\mathbf{x})=\underset{i}{\operatorname{argmin}}\left(\left\|I(\mathbf{x})-c_{i}\right\|^{2}\right) .
$$

The iteration stops if either the maximum iteration number is reached or the resolution is stable, i.e. $\max _{i \mathbf{x}}\left\{\left|u_{i}(\mathbf{x})^{(n)}-u_{i}(\mathbf{x})^{(n-1)}\right|\right\}<\epsilon$ where $\epsilon$ is a predetermined termination criterion and $n$ is the current iteration step and $n-1$ the last iteration. It is obvious that the standard FCM classifies pixels independently without considering correlation among neighbouring pixels and it is therefore sensitive to noise and lacks the ability to deal with intensity inhomogeneity [20, 27].

\subsection{Bias Corrected Fuzzy C-Means}

To overcome the drawbacks of FCM, Ahmed et al. proposed a modified FCM method to segment images with intensity inhomogeneity and correct the bias field at the same time [28]. They named the modified method bias corrected fuzzy c-means (BCFCM), in which a spatial constraint is introduced as a regularization term to allow the label of a pixel to be influenced by labels in its immediate neighbourhood. The neighbourhood effect can bias the solution toward to piecewise-homogeneous labels with the following modified objective function:

$$
\begin{aligned}
& F_{b c f c m}=\quad \int_{\Omega} \sum_{i=1}^{N}\left\|I_{\log _{a}}(\mathbf{x})-b_{\log _{a}}(\mathbf{x})-c_{i}\right\|^{2} u_{i}^{q}(\mathbf{x}) d \mathbf{x} \\
& +\frac{\alpha}{N_{R}} \int_{\Omega} \sum_{i=1}^{N}\left(\int_{y \in N_{\mathbf{x}}}\left\|I_{\log _{a}}(\mathbf{y})-b_{\log _{a}}(\mathbf{y})-c_{i}\right\|^{2} d \mathbf{y}\right) u_{i}^{q}(\mathbf{x}) d \mathbf{x}
\end{aligned}
$$

where $I_{\log _{a}}(\mathbf{x})=J_{\log _{a}}(\mathbf{x})+b_{\log _{a}}(\mathbf{x})+n_{\log _{a}}(\mathbf{x})$ is a logarithmic transformation of Eq. (1) with base number $a, N_{\mathbf{x}}$ stands for the set of neighbouring pixels existing in the window around $\mathbf{x}$, and $N_{R}$ is the cardinality of $N_{\mathbf{x}}$.

This modified objective function $J_{b c f c m}$ can be minimized in a fashion similar to the standard FCM. For other variables fixed, we minimize the energy $F_{b c f c m}$ with respect to $c_{i}$ by resolving $\frac{\partial F_{b c f c m}}{\partial c_{i}}=0$ and obtain the minimizer

$$
\check{c}_{i}=\frac{\int_{\Omega} u_{i}^{q}(\mathbf{x})\left(\left(I_{\log }(\mathbf{x})-b_{\log }(\mathbf{x})\right)+\frac{\alpha}{N_{R}} \int_{y \in N_{\mathbf{x}}}\left(I_{l o g}(\mathbf{y})-b_{\log }(\mathbf{y})\right) d \mathbf{y}\right) d \mathbf{x}}{(1+\alpha) \int_{\Omega} u_{i}^{q}(\mathbf{x}) d \mathbf{x}}, \quad i=1,2, \ldots, N .
$$

For other variables fixed, we minimize the energy $F_{b c f c m}$ with respect to $u_{i}(\mathbf{x})$ subject to the constraint that $\sum_{i=1}^{N} u_{i}(\mathbf{x})=1$ using Lagrange multiplier method. For the case $q>1$, the energy functional $F_{b c f c m}$ is minimized by $\tilde{u}_{i}(\mathbf{x})$, given by

$$
\check{u}_{i}(\mathbf{x})=\frac{\left(\left\|I_{l o g}(\mathbf{x})-b_{l o g}(\mathbf{x})-c_{i}\right\|^{2}+\frac{\alpha}{N_{R}} \int_{y \in N_{\mathbf{x}}}\left(\left\|I_{l o g}(\mathbf{y})-b_{l o g}(\mathbf{y})-c_{i}\right\|^{2}\right) d \mathbf{y}\right)^{\frac{1}{1-q}}}{\sum_{j=1}^{N}\left(\left\|I_{l o g}(\mathbf{x})-b_{l o g}(\mathbf{x})-c_{j}\right\|^{2}+\frac{\alpha}{N_{R}} \int_{y \in N_{\mathbf{x}}}\left(\left\|I_{l o g}(\mathbf{y})-b_{l o g}(\mathbf{y})-c_{j}\right\|^{2}\right) d \mathbf{y}\right)^{\frac{1}{1-q}}}, \quad i=1,2, \ldots, N .
$$


For $q=1$, it can be shown that the minimizer $\check{u}_{i}(\mathbf{x})$ is given by

$$
\check{u}_{i}(\mathbf{x})=\left\{\begin{array}{ll}
1, & i=i_{\text {min_bbfcm }}(\mathbf{x}) \\
0, & i \neq i_{\text {min_bcfcm }}(\mathbf{x})
\end{array} \quad i=1,2, \ldots, N\right.
$$

where

$$
i_{m i n \_b c f c m}(\mathbf{x})=\underset{i}{\operatorname{argmin}}\left(\left\|I_{l o g}(\mathbf{x})-b_{l o g}(\mathbf{x})-c_{i}\right\|^{2}\right) .
$$

For other variables fixed, we minimize the energy $F_{b c f c m}$ with respect to $b_{l o g}(\mathbf{x})$ by resolving $\frac{\partial F_{b c f c m}}{\partial b_{l o g}(\mathbf{x})}=0$ and obtain the minimizer

$$
\check{b}_{l o g}(\mathbf{x})=I_{l o g}(\mathbf{x})-\frac{\sum_{i=1}^{N} u_{i}^{q}(\mathbf{x}) c_{i}}{\sum_{i=1}^{N} u_{i}^{q}(\mathbf{x})} .
$$

Remark 1: It is obvious that the objective function of BCFCM is defined based on mapping the multiplicative bias field to an additive one with logarithmic transformation. As shown in Fig. 2, range of the transformed intensities is much more contracted than original intensities. This will inevitably reduce intensity differences of different tissues and will affect behaviours of the clustering algorithm negatively especially if the original image is noisy and inhomogeneous in intensity [32]. Furthermore, BCFCM is too time consuming due to the introduction of spatial constraint term and is not robust enough to tolerate noise and outliers as pointed out by Chen and Zhang in [29]. They therefore proposed a modified method to improve its time performance in [29], but their method lacks the ability to correct the bias field from images with intensity inhomogeneity.
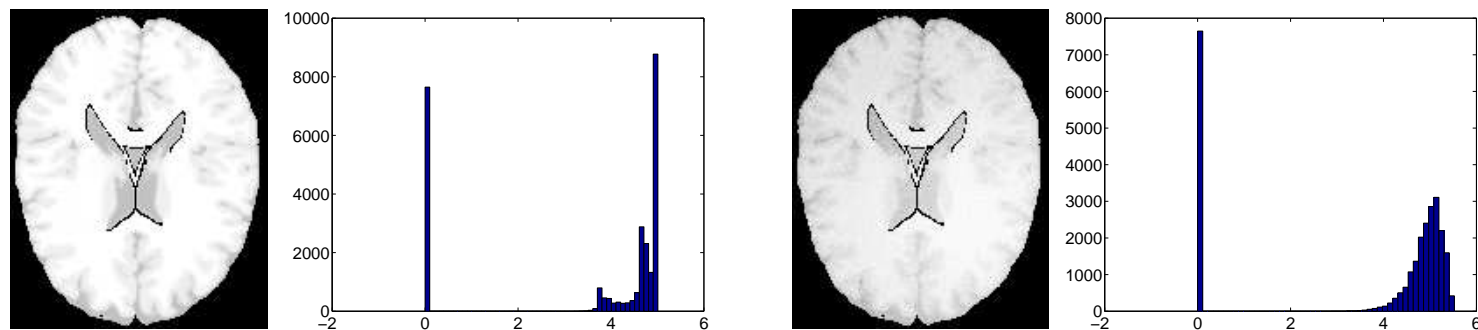

Figure 2: Appearance and intensity histograms of the images used in Fig. 1 after intensities of the images have been logarithmicly transformed. The left and right images are corresponding to the left and right ones in Fig. 1 respectively.

\subsection{Multiplicative Intrinsic Component Optimization}

To segment images with intensity inhomogeneity fast and correct the bias field simultaneously, Li et al. proposed a multiplicative intrinsic component optimization (MICO) model in [31]. In MICO, the slowly and smoothly varying property of the bias field $b$ is ensured by a linear combination of a given set of smooth basis functions $g_{1}, g_{2}, \ldots$, and $g_{M}$ with weighting coefficients $w_{1}, w_{2}, \ldots, w_{M}$, i.e.,

$$
b(\mathbf{x})=\mathbf{w}^{T} G(\mathbf{x})
$$


where $(\cdot)^{T}$ is the transpose operator, and $\mathbf{w}$ and $G(\mathbf{x})$ are column vectors defined by $\mathbf{w}=\left(w_{1}, w_{2}, \ldots, w_{M}\right)^{T}$ and $G(\mathbf{x})=\left(g_{1}(\mathbf{x}), g_{2}(\mathbf{x}), \ldots, g_{M}(\mathbf{x})\right)^{T}$, respectively.

In MICO, the following objective function is minimized to simultaneously segment a image with intensity inhomogeneity and estimate the bias field as described in 31]:

$$
F_{\text {mico }}(\mathbf{u}, \mathbf{c}, \mathbf{w})=\int_{\Omega} \sum_{i=1}^{N}\left\|I(\mathbf{x})-\mathbf{w}^{T} G(\mathbf{x}) c_{i}\right\|^{2} u_{i}^{q}(\mathbf{x}) d \mathbf{x}
$$

where $q \geq 1$, and $\mathbf{c}$ and $\mathbf{u}$ are column vectors defined by $\mathbf{c}=\left(c_{1}, c_{2}, \ldots, c_{N}\right)^{T}$ and $\mathbf{u}=\left(u_{1}, u_{2}, \ldots, u_{N}\right)^{T}$, respectively.

For fixed $\mathbf{w}$ and $\mathbf{u}$, the energy $F(\mathbf{u}, \mathbf{c}, \mathbf{w})$ can be minimized with respect to $\mathbf{c}$ by successively resolving $\frac{\partial F_{\text {mico }}}{\partial c_{i}}=0, i=1,2, \ldots, N$. Thus, the energy function $F(\mathbf{u}, \mathbf{c}, \mathbf{w})$ is minimized at $\hat{\mathbf{c}}=\left(\hat{c}_{1}, \hat{c}_{2}, \ldots, \hat{c}_{N}\right)^{T}$ with

$$
\hat{c}_{i}=\frac{\int_{\Omega} I(\mathbf{x}) b(\mathbf{x}) u_{i}^{q}(\mathbf{x}) d \mathbf{x}}{\int_{\Omega} b^{2}(\mathbf{x}) u_{i}^{q}(\mathbf{x}) d \mathbf{x}}, \quad i=1,2, \ldots, N
$$

For fixed $\mathbf{c}$ and $\mathbf{u}$, the energy $F(\mathbf{u}, \mathbf{c}, \mathbf{w})$ can be minimized with respect to $\mathbf{w}$ by resolving $\frac{\partial F_{\text {mico }}}{\partial \mathbf{w}}=\mathbf{0}$ where $\mathbf{0}$ is a constant function with value 0 . Thus, the optimal $\hat{\mathbf{w}}=\left(\hat{w}_{1}, \hat{w}_{2}, \ldots, \hat{w}_{N}\right)^{T}$ that minimizes $F(\mathbf{u}, \mathbf{c}, \mathbf{w})$ is given by

$$
\hat{\mathbf{w}}=\left(\int_{\Omega} G(\mathbf{x}) G^{T}(\mathbf{x})\left(\sum_{i=1}^{N} c_{i}^{2} u_{i}^{q} \mathbf{x}\right) d \mathbf{x}\right)^{-1} \int_{\Omega} G(\mathbf{x}) I(\mathbf{x})\left(\sum_{i=1}^{N} c_{i} u_{i}^{q} \mathbf{x}\right) d \mathbf{x} .
$$

For fixed $\mathbf{w}$ and $\mathbf{c}$, the energy $F(\mathbf{u}, \mathbf{c}, \mathbf{w})$ can be minimized with respect to $\mathbf{u}=\left(u_{1}, u_{2}, \ldots, u_{N}\right)^{T}$ subject to the constraint that $\sum_{i=1}^{N} u_{i}(\mathbf{x})=1$. For the case $q>1$, the energy functional $F(\mathbf{u}, \mathbf{c}, \mathbf{w})$ is minimized at $\hat{\mathbf{u}}=\left(\hat{u}_{1}, \hat{u}_{2}, \ldots, \hat{u}_{N}\right)^{T}$, given by

$$
\hat{u}_{i}(\mathbf{x})=\frac{\left(\left\|I(\mathbf{x})-\mathbf{w}^{T} G(\mathbf{x}) c_{i}\right\|^{2}\right)^{\frac{1}{1-q}}}{\sum_{j=1}^{N}\left(\left\|I(\mathbf{x})-\mathbf{w}^{T} G(\mathbf{x}) c_{j}\right\|^{2}\right)^{\frac{1}{1-q}}} \quad i=1,2, \ldots, N .
$$

139 For $q=1$, it can be shown that the minimizer $\hat{\mathbf{u}}=\left(\hat{u}_{1}, \hat{u}_{2}, \ldots, \hat{u}_{N}\right)^{T}$ is given by

$$
\hat{u}_{i}(\mathbf{x})=\left\{\begin{array}{ll}
1, & i=i_{\text {min_mico }}(\mathbf{x}) \\
0, & i \neq i_{\text {min_mico }}(\mathbf{x})
\end{array} \quad i=1,2, \ldots, N\right.
$$

where

$$
i_{\text {min_mico }}(\mathbf{x})=\underset{i}{\operatorname{argmin}}\left(\left\|I(\mathbf{x})-\mathbf{w}^{T} G(\mathbf{x}) c_{i}\right\|^{2}\right) .
$$

Remark 2: As the energy function $F_{\text {mico }}(\mathbf{u}, \mathbf{c}, \mathbf{w})$ is convex in each of its variables, MICO is able to 
simultaneously segment images with intensity inhomogeneity and correct the bias field [31]. But it is obvious that MICO is a pixel-based clustering method and is therefore sensitive to noise.

\section{The Proposed Framework}

To segment images with both noise and intensity inhomogeneity and correct the bias field simultaneously, a framework is proposed in this section. As shown in Fig. 3, the proposed framework consists of two primary constituent parts: non-local means denoising (NLMD) and bias correction embedded FCM (BCEFCM). In the proposed framework, noise in the interested image is first removed by CUDA accelerated nonlocal means denoising. Then, BCEFCM is used to segment the noise removed image and correct the bias field. More details about the proposed image segmentation and bias correction framework are described in the following subsections.

Remark 3: The main difference between the proposed method and BCFCM is that no additional terms are introduced to the objective function of FCM except that the bias field is considered as a multiplier of cluster centers. In addition, the CUDA accelerated non-local denoising algorithm is robust enough to tolerate noise and outliers.

\subsection{Non-local Means Denoising}

In order to accurately segment images described using the image model given in Eq. (1), effective noise reduction is necessary. However, traditional denoising methods may blur the image especially at boundaries because they generally replace intensity of a given pixel with an average of its neighbouring pixels, which therefore increases the difficulty of segmenting the image correctly [33]. In fact, there is no reason for the most similar pixels to a given pixel to be close to it [34]. Thus, Buades et al. proposed a non-local means denoising (NLMD) method to reduce noise from noisy images by averaging similar pixels in the whole image domain not only in the neighbourhood of a given pixel, which reserves local characteristics of the image and will not blur the image [35].

For an observed noisy image $I$, the estimated value $N L(I)(\mathbf{x})$, for a given pixel $\mathbf{x} \in \Omega$, is computed in NLMD as a weighted average of all the pixels in the image domain $\Omega$, i.e.,

$$
N L(I)(\mathbf{x})=\frac{1}{\int_{\Omega} W(\mathbf{x}, \mathbf{y}) d \mathbf{y}} \int_{\Omega} W(\mathbf{x}, \mathbf{y}) I(\mathbf{y}) d \mathbf{y}
$$

where $W(\mathbf{x}, \mathbf{y})$ is the weighting values assigned to $I(\mathbf{y})$ in the restoration of pixel $\mathbf{x}$ depending on the similarity of pixel $\mathbf{y}$ to $\mathbf{x}$. Let $G_{\delta}$ be the Gaussian function with standard deviation $\delta$, we define

$$
\left(G_{\delta} *|I(\mathbf{x}+.)-I(\mathbf{y}+.)|^{2}\right)(0)=\int_{\Omega} G_{\delta}(\mathbf{t})|I(\mathbf{x}+\mathbf{t})-I(\mathbf{y}+\mathbf{t})|^{2} d \mathbf{t}
$$



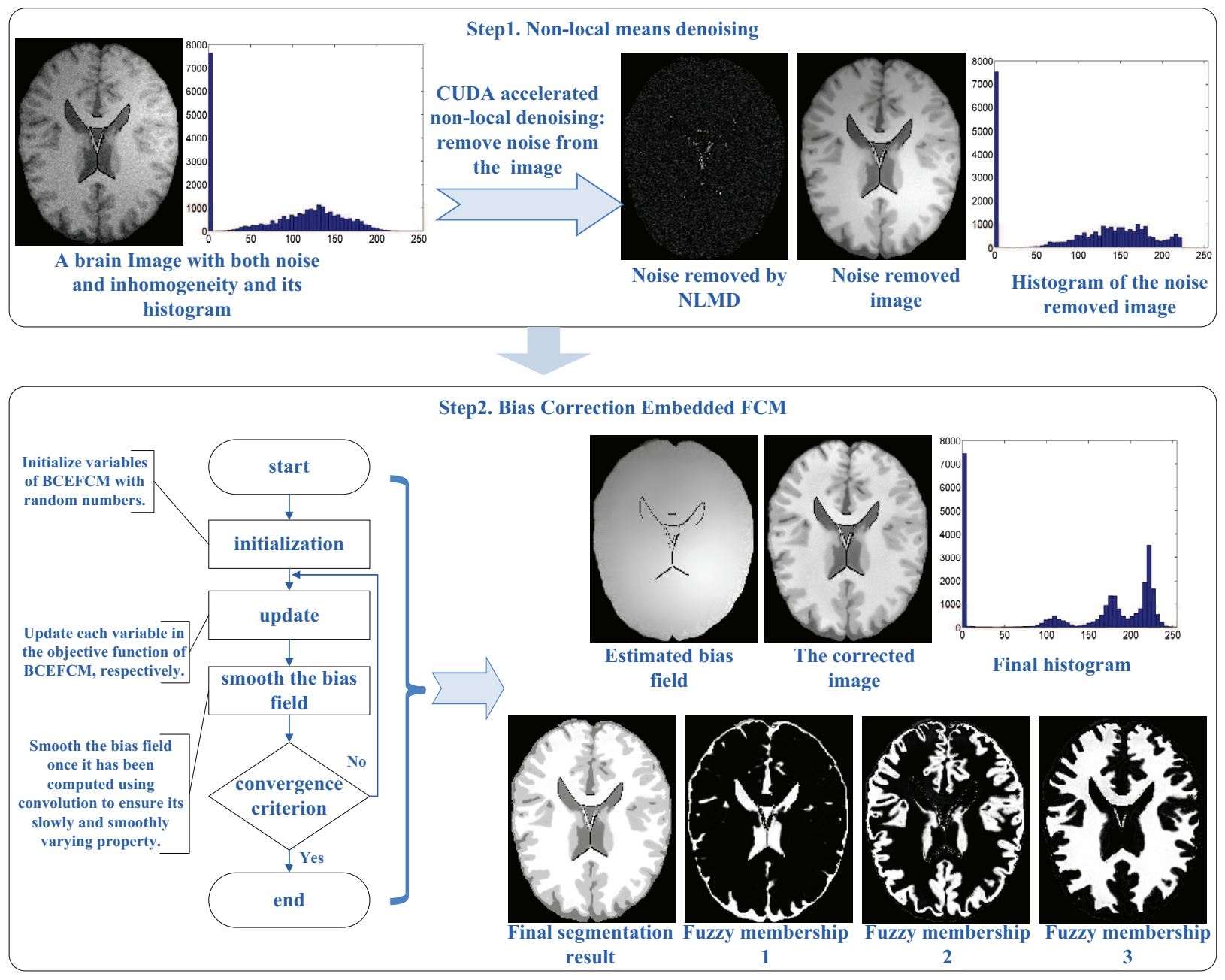

Figure 3: The proposed image segmentation framework of NLMD+BCEFCM

and therefore

$$
W(\mathbf{x}, \mathbf{y})=e^{\frac{-\left(G_{\delta} *|I(\mathbf{x}+\cdot)-I(\mathbf{y}+.)|^{2}\right)(0)}{h^{2}}}
$$

where $h$ acts as a filtering parameter.

The equation (21) indicates that $I(\mathbf{x})$ is replaced by a weighted average of $I(\mathbf{y})$. The weightings are effective only if intensities in the Gaussian window around $\mathbf{y}$ are similar to those in the corresponding window around $\mathbf{x}$. Thus, NLMD takes advantage of image self-similarity to reduce noise and therefore the local characteristics of the image can be preserved as shown in Fig. 4. It can also be seen from Fig. 4 that the intensity inhomogeneity still exists in the noise removed image and therefore it is still hardly to cluster the intensities into different classes due to the intensity overlaps as seen from the histogram of the noise removed image. 

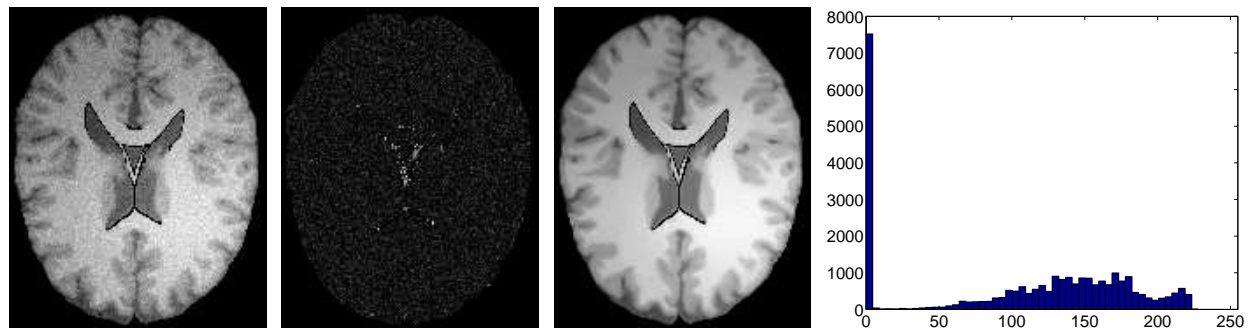

Figure 4: Results of NLMD on the image used in Fig. 1 1 with both noise and intensity inhomogeneity. From left to right: the original image, noise removed by NLMD, noise-removed image, and the histogram of the noise-removed image.

\subsection{Non-local Means Denoising Accelerated With CUDA}

From Eq. (21), it can been seen that the original definition of NLMD considers that each pixel of a given image can be linked to all the others. But pixels that are taken into account in the weighted average are practically restricted in a neighbourhood with size of $(2 M+1)^{m}$ centered at a given pixel $\mathbf{x}$ where $m$ is the dimensionality of the image [36]. The neighbourhood is name as search window. Similarity of each pixel in the search window to the given pixel $\mathbf{x}$ depends on the closeness of intensities in their local neighbourhoods with size of $(2 d+1)^{m}$. It is obvious that the main disadvantage of NLMD is the computational burden due to its complexity in the order of $O\left(\left(N(2 M+1)^{m}(2 d+1)^{m}\right)\right)$ for images with $N$ pixels. As Coupé et al. mentioned in [37], for a three dimensional image with resolution $181 \times 217 \times 181$, computational time of NLMD reaches up to 6 hours to reduce noise from the image with parameters $M=5$ and $d=1$ which is far beyond a reasonable duration expected for a denoising algorithm in practice.

Due to its ever-increasing in computing power and powerful capabilities in general purpose computing power, the graphics processing unit (GPU) has been widely used to accelerate image processing methods [38]. In particular, Compute Unified Device Architecture (CUDA) has been introduced by NIVIDA in which the GPU is regarded as a parallel computation device. Device instructions are encapsulated into a kernel function which is written with language $\mathrm{C}$ and invoked by the CPU [39]. But the instructions are finally executed on the GPU with thousands of threads executing simultaneously at the same time [40]. Therefore, data-parallel computation can be easily implemented with standard C language as a general purpose computation on GPUs that are CUDA enabled [41].

To improve the time performance of NLMD, we tried to accelerate it with CUDA as described in Algorithm 1. Unfortunately, there is a watchdog timer for triggering a system reset if the kernel function occupies the GPU longer than 5-15 seconds which may be different depending on the specific setting of the operating system. Readers are referred to http://msdn.microsoft.com/en-us/library/ms856964 for more details. Therefore, in our implementation, CUDA threads are created based on the resolution of the input image for small burden of launch and loading parameters. If the watchdog time reaches for larger search windows and/or neighbourhoods, we split the kernel function into $(2 M+1)^{m}$ smaller kernel functions. If the watchdog time reaches again (hardly happens), the kernel functions are further split into $(2 d+1)^{m}$ 
smaller kernels. In this way, we obtain a 200 times speedup by accelerating NLMD on Geforce GT310 (45 seconds) than its implementation on intel i5 processor (2 hours and a half) for de-nosing a $181 \times 217 \times 181$ image with $M=2$ and $d=1$. It is obvious that the split of kernel function will introduce more function launch and invocation if watchdog time reaches. Thus, the speedup ratio reduces to 150 (less than 3 minutes) for de-noising the image with resolution of $181 \times 217 \times 181$ with $M=5$ and $d=1$.

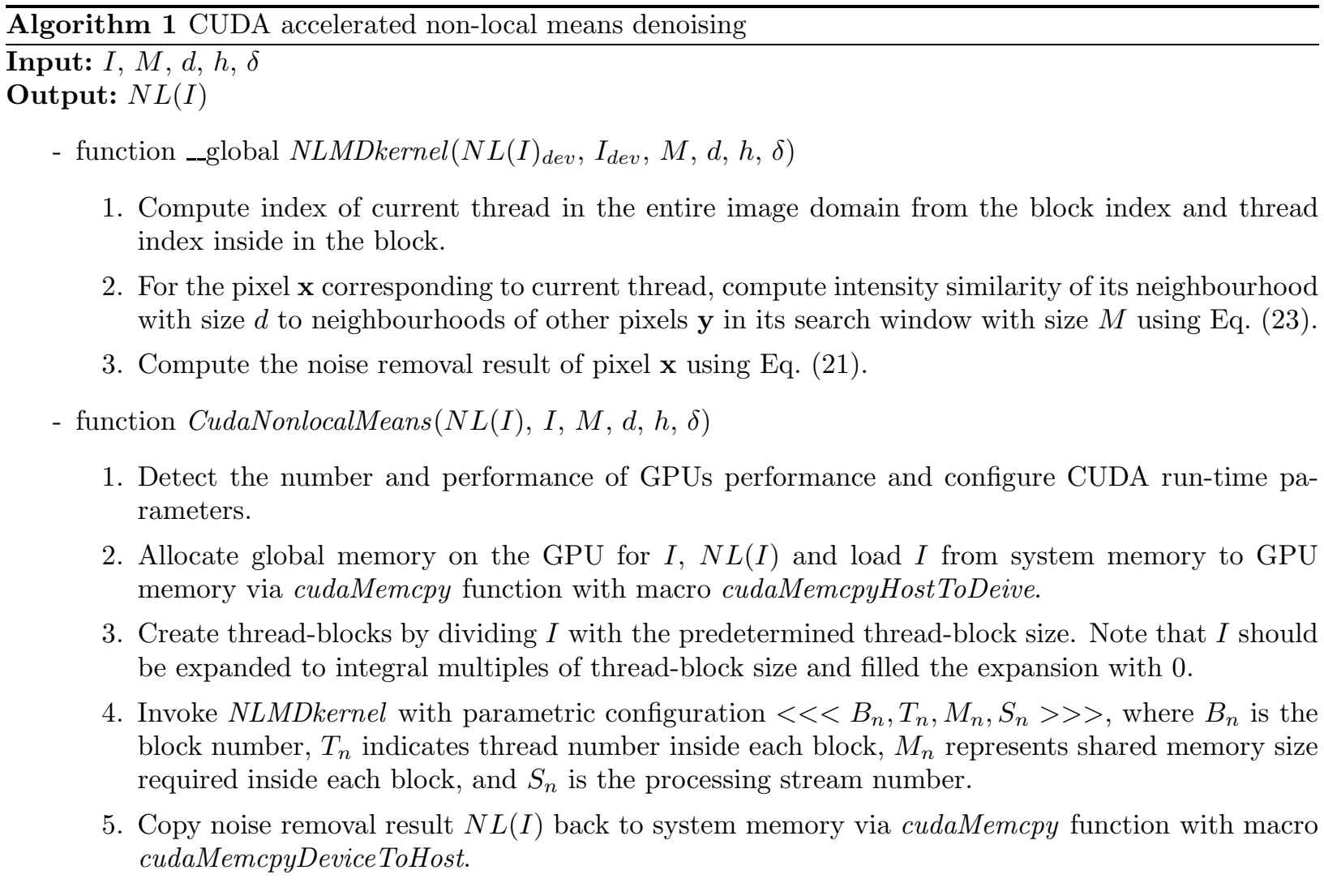

\subsection{Bias Correction Embedded Fuzzy C-Means}

After noise reduction using NLMD, the image model represented by Eq. (11) can be rewritten as

$$
N L(I)(\mathbf{x})=b(\mathbf{x}) J(\mathbf{x})
$$

As mentioned earlier, the true image $J$ is assumed to be piecewise constant and therefore takes a specific intensity $c_{i}$ for the $i$-th type of objects in the region $\Omega_{i}$. Thus, if $\mathbf{x}$ is most probably classified as the $i$-th type of objects, then the intensity of the true image $J$ at pixel $\mathbf{x}$ is close to the constant $c_{i}$, i.e.

$$
J(\mathbf{x}) \approx c_{i} \quad \text { for } \quad \mathbf{x} \in \Omega_{\mathrm{i}} .
$$


Then, in view of the image model in Eq. (24), we have

$$
N L(I)(\mathbf{x}) \approx b(\mathbf{x}) c_{i} \quad \text { for } \quad \mathbf{x} \in \Omega_{\mathrm{i}} .
$$

Therefore, the intensities in the set

$$
N L(I)_{i}=\left\{N L(I)(\mathbf{x}): \mathbf{x} \in \Omega_{i}\right\}
$$

form a cluster with the cluster centroid $m_{i} \approx b(\mathbf{x}) c_{i}$. This clustering property indicates that the intensities in the image domain $\Omega$ can be classified into $N$ clusters with centroids $m_{1} \approx b(\mathbf{x}) c_{1}, m_{2} \approx b(\mathbf{x}) c_{2}, \ldots$, and $m_{N} \approx b(\mathbf{x}) c_{N}$, respectively. To classify these intensities, we define

$$
F_{b c e f c m}=\int_{\Omega} \sum_{i=1}^{N}\left\|N L(I)(\mathbf{x})-b(\mathbf{x}) c_{i}\right\|^{2} u_{i}^{q}(\mathbf{x}) d \mathbf{x} .
$$

To generalize the above defined formulation and provide an option for users to improve the segmentation results, we introduce positive weighting coefficients $\lambda_{1}, \lambda_{2}, \ldots, \lambda_{N}$ for the $N$ clusters and modified the above energy as follows

$$
F_{b c e f c m}=\int_{\Omega} \sum_{i=1}^{N} \lambda_{i}\left\|N L(I)(\mathbf{x})-b(\mathbf{x}) c_{i}\right\|^{2} u_{i}^{q}(\mathbf{x}) d \mathbf{x} .
$$

Remark 4: It is obvious that the objective function $F_{b c e f c m}$ defined in Eq. (28) is a special case of $F_{\text {bcefcm }}$ in Eq. (29) with $\lambda_{i}=1$ for $i=1,2, \ldots, N$. It is worth further pointing out that if we replace the noise removed image $N L(I)(\mathbf{x})$ with the original image $I(\mathbf{x})$, the proposed objective function of BCEFCM defined in Eq. (29) reduces to the objective function of FCM defined in Eq. (2) when the bias field is a constant function 1, i.e., $b(\mathbf{x})=1$ for $\mathbf{x} \in \Omega$. That is to say the proposed BCEFCM described in Eq. (29) is a generalization of the well-known FCM given in Eq. (2). Note that for all other parameters fixed, the smaller the parameter $\lambda_{i}$ is, the greater the $i$-cluster is, and vice versa. We will discuss the impact of parameter $\lambda_{i}$ on the size of the $i$-th cluster in section 5 .

\subsection{Energy Minimization}

Energy minimization of $F_{b c e f c m}$ can be achieved by alternately minimizing it with respect to each of its variables. For fixed $b(\mathbf{x})$ and $u_{i}(\mathbf{x}), i=1,2, \ldots, N$, we minimize $F_{b c e f c m}$ with respect to $c_{i}$ by resolving $\frac{\partial F_{b c e f c m}}{\partial c_{i}}=0$. It is obvious that $F_{b c e f c m}$ is minimized at $c_{i}=\breve{c}_{i}$, given by

$$
\breve{c}_{i}=\frac{\int_{\Omega} b(\mathbf{x}) N L(I)(\mathbf{x}) u_{i}^{q}(\mathbf{x}) d \mathbf{x}}{\int_{\Omega} b^{2}(\mathbf{x}) u_{i}^{q}(\mathbf{x}) d \mathbf{x}} .
$$

For fixed $u_{i}(\mathbf{x})$ and $c_{i}, i=1,2, \ldots, N$, we minimize $F_{b c e f c m}$ with respect to $b(\mathbf{x})$ by resolving $\frac{\partial F_{b c e f c m}}{\partial b(\mathbf{x})}=0$. 
It can be shown that $F_{b c e f c m}$ is minimized at $b(\mathbf{x})=\breve{b}(\mathbf{x})$, given by

$$
\breve{b}(\mathbf{x})=\frac{N L(I)(\mathbf{x}) \sum_{i=1}^{N} \lambda_{i} c_{i} u_{i}^{q}(\mathbf{x})}{\sum_{i=1}^{N} \lambda_{i} c_{i}^{2} u_{i}^{q}(\mathbf{x})}
$$

For the case $q>1$, minimization of $F_{b c e f c m}$ with respect to $u_{i}(\mathbf{x})$ can be implemented by resolving the following Lagrangian equation:

$$
\sum_{i=1}^{N} \lambda_{i} \int_{\Omega}\left\|N L(I)(\mathbf{x})-b(\mathbf{x}) c_{i}\right\|^{2} u_{i}^{q}(\mathbf{x}) d \mathbf{x}-\lambda\left(\sum_{i=1}^{N} u_{i}(\mathbf{x})-1\right)=0
$$

where $\lambda$ is the Lagrangian multiplier and $\sum_{i=1}^{N} u_{i}(\mathbf{x})=1$ is the extremum condition. For fixed $b(\mathbf{x})$ and $c_{i}, i=1,2, \ldots, N$, we take partial derivative of the above equation with respect $u_{i}(\mathbf{x})$, set the result to 0 , and resolve the equations with the constraint that $\sum_{i=1}^{N} u_{i}(\mathbf{x})=1$. Then, it can be shown that $F_{b c e f c m}$ is minimized at $u_{i}(\mathbf{x})=\breve{u}_{i}(\mathbf{x})$, given by

$$
\breve{u}_{i}(\mathbf{x})=\frac{\left(\lambda_{i}\left\|N L(I)(\mathbf{x})-b(\mathbf{x}) c_{i}\right\|^{2}\right)^{\frac{1}{1-q}}}{\sum_{j=1}^{N}\left(\lambda_{j}\left\|N L(I)(\mathbf{x})-b(\mathbf{x}) c_{j}\right\|^{2}\right)^{\frac{1}{1-q}}}
$$

For the case $q=1$, it can be shown that the minimizer $\breve{u}_{i}(\mathbf{x})$ is given by

$$
\breve{u}_{i}(\mathbf{x})=\left\{\begin{array}{ll}
1, & i=i_{\text {min_bcefcm }}(\mathbf{x}) \\
0, & i \neq i_{\text {min_bcefcm }}(\mathbf{x})
\end{array} \quad i=1,2, \ldots, N\right.
$$

where

$$
i_{m i n \_b c e f c m}(\mathbf{x})=\underset{i}{\operatorname{argmin}}\left(\lambda_{i}\left\|N L(I)(\mathbf{x})-b(\mathbf{x}) c_{i}\right\|^{2}\right) .
$$

\subsection{Implementation}

To ensure the slowly and smoothly varying property of the bias filed, we convolute the bias field $b$ with a kernel function $K$ in the implementation to smooth it as soon as it has been updated using Eq. (31) in each iteration. Note that the choice of the convolution kernel $K$ is important but flexible as long as it is a non-negative function defined on $\Omega$ with range of $[0,+\infty)]$ and satisfies:

1) $K(-\mathbf{x})=K(\mathbf{x})$;

2) $K(\mathbf{x})>K(\mathbf{y})$, if $|\mathbf{x}|<|\mathbf{y}|$, and $\lim _{|\mathbf{x}| \rightarrow+\infty} K(\mathbf{x})=0$;

3) $\int_{\Omega} K(\mathbf{x}) d \mathbf{x}=1$.

In this paper, the following averaging filter with size of $\rho$ is chosen as the convolution kernel function $K$ :

$$
K_{\rho}(\mathbf{u})=\left\{\begin{array}{lll}
a, & \text { for } & |\mathbf{u}| \leq \rho \\
0, & \text { for } & |\mathbf{u}|>\rho
\end{array}\right.
$$


where $a>0$ is a normalization factor such that $\int_{|\mathbf{u}| \leq \rho} K_{\rho}(\mathbf{u})=1$.

The implementation of BCEFCM can be straightforwardly expressed as follows.

- Step 1. Initialize $\mathbf{u}=\left(u_{1}, u_{2}, \ldots, u_{N}\right)$ and $\mathbf{c}=\left(c_{1}, c_{2}, \ldots, c_{N}\right)$.

- Step 2. Update $b(\mathbf{x})$ as $\breve{b}(\mathbf{x})$ in Eq. (31) for $\mathbf{x} \in \Omega$.

- Step 3. Convolute $b$ with kernel $K$ to smooth it.

- Step 4. Update $c_{i}$ as $\breve{c}_{i}$ in Eq. (30) for $i=1,2, \ldots, N$.

- Step 5. Update $u_{i}(\mathbf{x})$ as $\breve{u}_{i}(\mathbf{x})$ for $i=1,2, \ldots, 3$ and $\mathbf{x} \in \Omega$ in Eq. (33) if $q>1$ or in Eq. (34) if $q=1$.

- Step 6. Check convergence criterion and iteration number. If convergence has been reached or the iteration number exceeds a predetermined maximum number, stop the iteration, otherwise, go to Step 2.

The convergence criterion used in Step 6 is $\max _{i \mathbf{x}}\left\{\left|u_{i}(\mathbf{x})^{(n)}-u_{i}(\mathbf{x})^{(n-1)}\right|\right\}<\epsilon$ where $\epsilon$ is a predetermined termination criterion and $u_{i}(\mathbf{x})^{(n)}$ and $u_{i}(\mathbf{x})^{(n-1)}$ are the membership function $u_{i}(\mathbf{x})$ updated using Step 5 in the $n$-th and $n-1$-th iterations. Note that the convergence criterion of the proposed BCEFCM is usually reached in less than 20 iterations. Therefore, we set the maximum iteration number as 20 .

Remark 5: Although we initialize $\mathbf{u}=\left(u_{1}, u_{2}, \ldots, u_{N}\right)$ and $\mathbf{c}=\left(c_{1}, c_{2}, \ldots, c_{N}\right)$ and update $b$, $\mathbf{c}$, and $\mathbf{u}$ successively in our implementation, the initialization and update of each variable are flexible in fact as long as it is ensured that they are all updated once in each iteration and the update of $b$ is followed by smoothing the bias field with convolution.

\section{Experimental Results}

The proposed framework (NLMD+BCEFCM) has been extensively tested on both synthetic images and real images in Matlab R2011b on a computer with Intel(R) Core(TM) i5-3230M 2.6 GHz CPU, 4 GB RAM, and Windows 7 64-bit operating system. In this section, we first demonstrate the effectiveness of NLMD+BCEFCM in segmenting images with both noise and intensity inhomogeneity and the capability of BCEFCM in estimating the bias field on five distinctive images. We then qualitatively compare NLMD+BCEFCM with BCEFCM and three state-of-the-art methods on another five images. We finally apply NLMD+BCEFCM to Brainweb and IBSR datasets and compare segmentation accuracy of the proposed framework with BCEFCM and the state-of-the-art methods on the datasets quantitatively. For images used in this paper, we initialized $\mathbf{u}=\left(u_{1}, u_{2}, \ldots, u_{N}\right)$ and $\mathbf{c}=\left(c_{1}, c_{2}, \ldots, c_{N}\right)$ with random numbers. Unless otherwise specified, we set $M=7, d=3, h=10.0, \rho=7, q=2, \epsilon=0.01$, and $\lambda_{1}=\lambda_{2}=\ldots=\lambda_{N}=1$ in this paper. 


\subsection{Effectiveness of The Proposed Framework}

To demonstrate effectiveness of the proposed framework in noise remove, bias field correction, and image segmentation, we first applied it to a real MR noisy image of brain with also severe intensity inhomogeneity and show the results in Fig. 5. It can be seen from the original image shown in Fig. 5)(a) that the noise and bias field only affect foreground of the image, which consists of white matter (WM), grey matter (GM), and cerebrospinal fluid (CSF). From the histogram of the original image as shown in Fig. 5 (f), we can see that intensity ranges of WM, GM, and CSF in the image are overlapped which indicates that it is difficult to classify intensities of this image into WM, GM, and CSF straightforwardly. As shown in Fig. 5 (b), noise removed by NLMD are discrete and do not contain any structural information of the brain. Therefore, the noise removed image is much more noise free with its quality unaffected by NLMD even at boundaries of two different tissues as shown in Fig. 5(c). But it is still hard to segment the noise removed image into WM, GM, and CSF because of survival of the bias field. The remaining bias field maintains the overlap of intensity distribution between different tissues, which can be seen from the histogram of the noise removed image as shown in Fig. 5 (g). To segment the image correctly and estimate the bias field from the image simultaneously, we applied BCEFCM to the noise removed image and give the results in Fig. 5(d) and Fig. 5(i). Meanwhile, the bias field corrected image and its histogram are given in Fig. 5(e) and Fig. 5(h), respectively, from which we can see that the corrected image is close to be piecewise constant. On one hand, from the final segmentation results and fuzzy membership functions as shown in Fig. 5(i), Fig. 5(j), Fig. 5(k), and Fig. $5(1)$, we can see that the WM, GM, and CSF are well segmented. On the other hand, improvements of the image quality in terms of intensity homogeneity can not only be seen from the bias corrected image but also be demonstrated by comparing the histogram of the original image with the histogram of the bias corrected image as shown in Fig. 5(f) and Fig. 5(h). It is obvious that there are not well-separated peaks in histograms of the original image due to the mixture of intensity distribution caused by the bias field, but there are four well-defined and well-separated peaks in histograms of the bias corrected image, each corresponding to one tissue or the background. This demonstrates the capability of BCEFCM in correcting bias fields from images with intensity inhomogeneity.

To further demonstrate correctness of the proposed framework, we applied NLMD+BCEFCM to another four distinctive images and show the results in Fig. 6. The first image is a synthetic image with a bright heart-shaped object in the center of a darker circle. The entire image is filled with both noise and intensity inhomogeneity. The second image is a real image with ten coins, intensities of which are inhomogeneous because of the lighting. The third image is an X-ray image of a hand with severe intensity inhomogeneity. The fourth image used in this experiment is a real image of a leaf with irregular boundaries and slender petioles, which may be miss-segmented due to the existing of noise and intensity inhomogeneity. For the first image, parameter $N$ is set to be 3 , while $N=2$ for the others. The results of NLMD+BCEFCM on the four images are shown in four rows in Fig. 6 with original images, noise removed, estimated bias fields, 


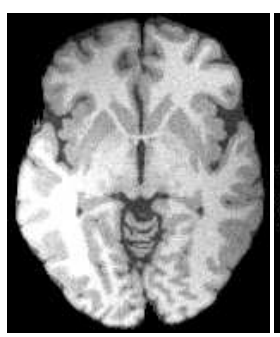

(a) Orignal image

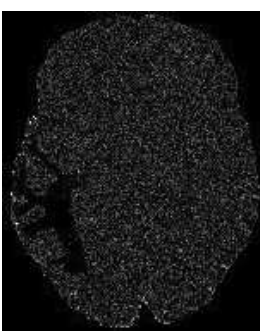

(b) Noise removed (c)

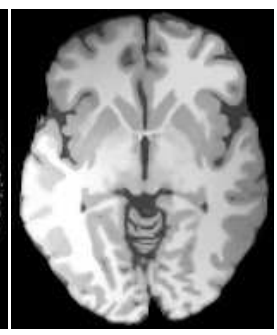

age
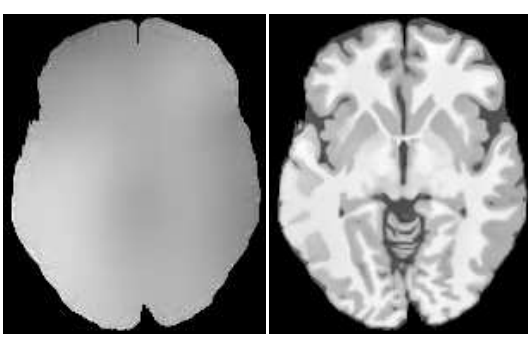

field rected image

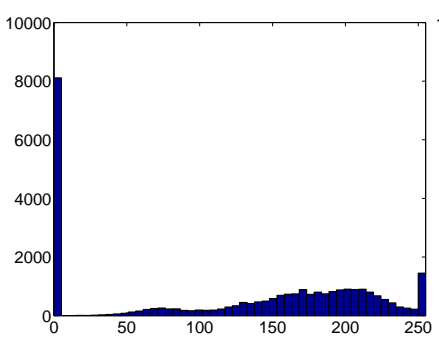

(f) Histogram of (a)

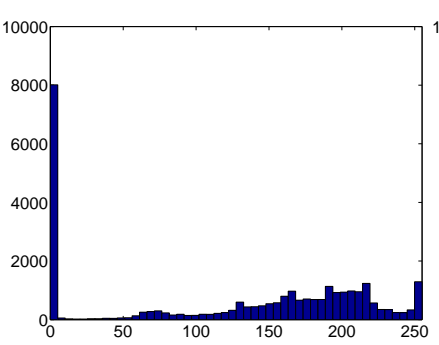

(g) Histogram of (c)

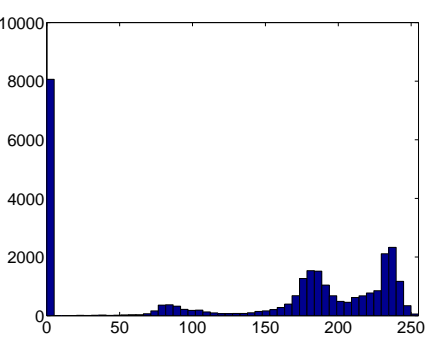

(h) Histogram of (e)

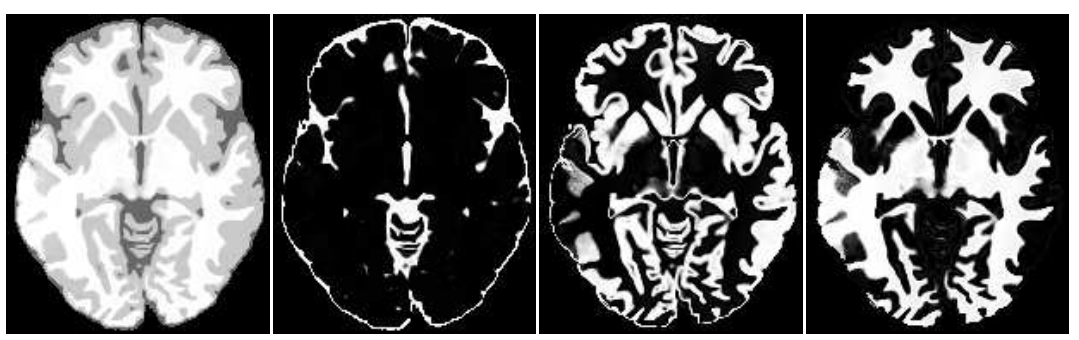

$\begin{array}{lccc}\text { (i) Segmentation } & \text { re-(j) Fuzzy membership(k) } & \text { Fuzzy } & \text { member-(l) Fuzzy membership } \\ \text { sult } & 1 & \text { ship } 2 & 3\end{array}$

Figure 5: Segmentation of a brain image with both noise and intensity inhomogeneity using NLMD+BCEFCM.

segmentation results, noise removed and bias field corrected images, and final intensity histograms given in six columns. It can be seen from Fig. [6 that 1) the noise removed and bias corrected images are much more noise-free and intensity homogeneous than the original images, 2) there are well-separated peaks in histograms of noise removed and bias corrected images, and 3) the images are all well segmented. Therefore, the proposed framework is effective in segmenting images with both noise and intensity inhomogeneity, removing the noise from the images, and correcting the bias field simultaneously.

\subsection{Qualitative Comparison with State-of-the Art Methods}

In this subsection, we compare the proposed framework (NLMD+BCEFCM) with FCM, BCFCM, MICO, and BCEFCM qualitatively eight distinctive images and give the results in Fig. (7)-Fig. (14), respectively. The first image is a synthetic image of rice-like objects with slight Gaussian additive noise and severe intensity inhomogeneity. The second image is also a synthetic image of three different geometrical shapes with both 

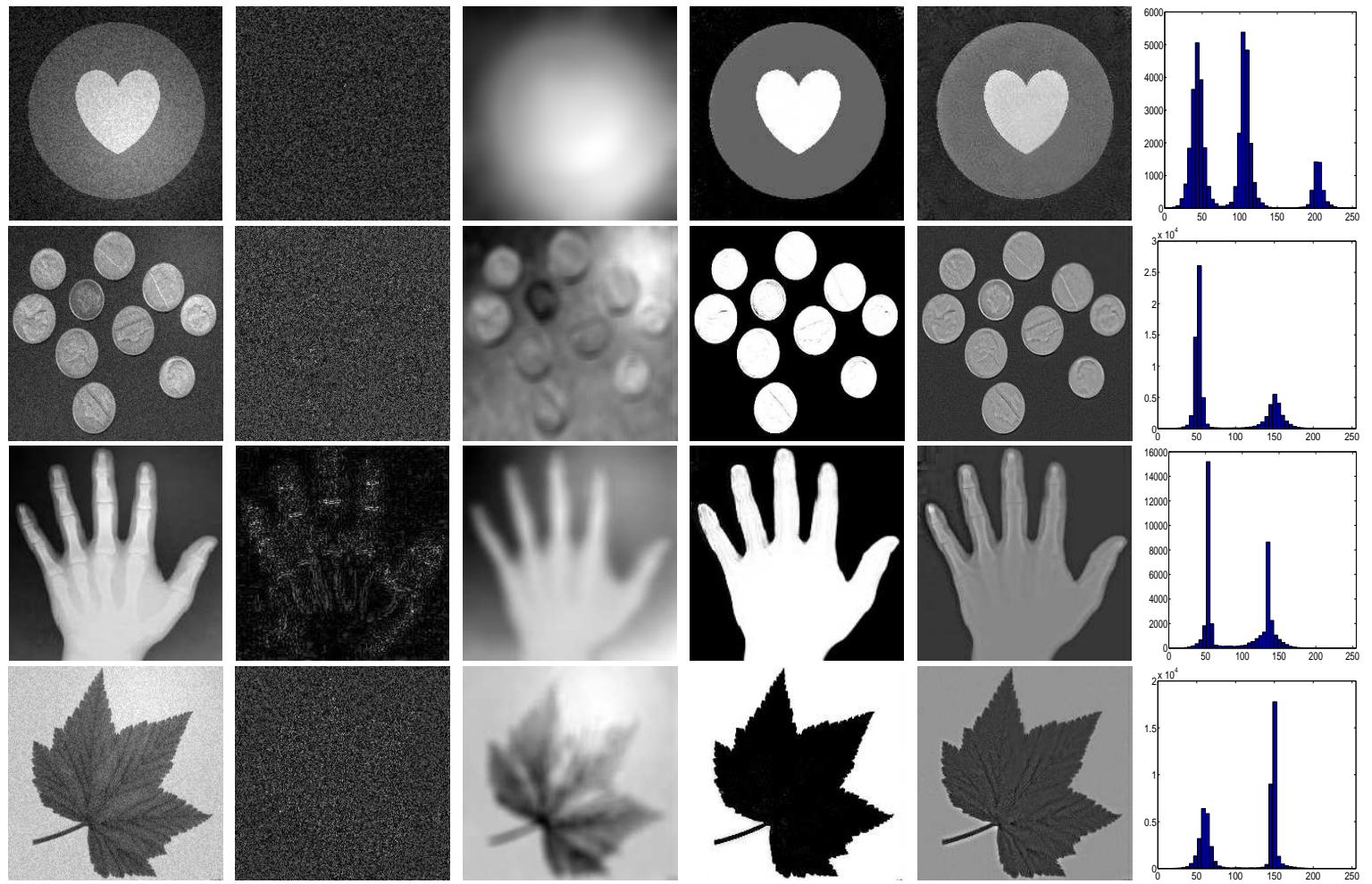

Figure 6: Results of NLMD+BCEFCM on one synthetic image and three real images shown in four rows. From left to right: original images, noise removed, estimated bias fields, segmentation results, noised removed and bias field corrected images, and final intensity histograms.

severe noise and intensity inhomogeneity. The third and fourth images are two real X-ray images of vessel with both noise and intensity inhomogeneity. The fifth image is a real MR image of brain with obvious noise and severe intensity inhomogeneity. Resolutions of the images are $256 \times 256,152 \times 152,111 \times 110$, $103 \times 131$, and $141 \times 157$, respectively. The rest three images are from The Berkeley Segmentation Dataset (BSD, http://wWw. eecs.berkeley.edu/Research/Projects/CS/vision/grouping/segbench/) [42].

In this experiment, we initialized cluster centroids with random numbers for FCM and updated membership functions and cluster centroids in turn in each iteration. For other methods, we initialized both cluster centroids and the bias field with random numbers and updated membership functions, cluster centroids, and the bias field in turn in each iteration. Parameter $\alpha$ of BCFCM was set to be 1 and the base number $a$ was set to be 1.02 in this experiment, while ten orthogonal three order Legendre polynomial functions were used to estimate the bias field for MICO. For the first five images used in this experiment, the original image, segmentation result of FCM, noise removed by NLMD, and energy curve of FCM are given in the first column of each figure. From the second to the fifth columns are the estimated bias fields, segmentation results, bias corrected images, and energy curves of BCFCM, MICO, BCEFCM, and NLMD+BCEFCM, respectively. 
From results on the first image as shown in Fig. [7 we can see that parts of the desired rice-like objects at bottom of the image are classified to background by FCM. Whereas, some pixels in the background at bottom of the image are considered as desired segmentation objects by BCFCM, MICO, and BCEFCM. Moreover, there are obvious noise at bottom of the bias corrected images obtained from BCFCM and BCEFCM. It is obvious that NLMD+BCEFCM achieved the best segmentation result and bias corrected image. Furthermore, from the energy curves as show in the bottom of Fig. 7, we can see that objective function of BCEFCM converged is less than 15 iterations which is close to the others.
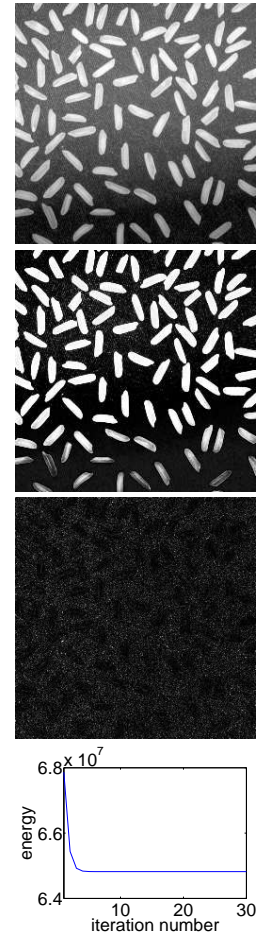
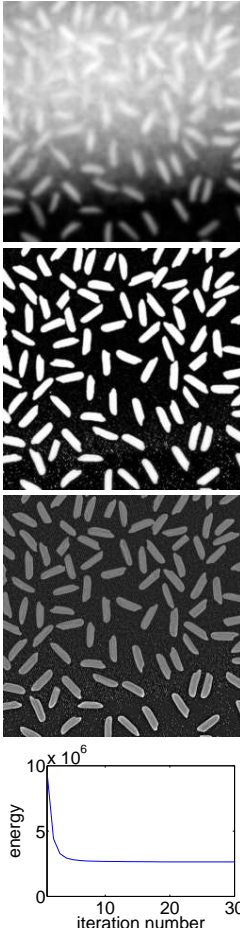
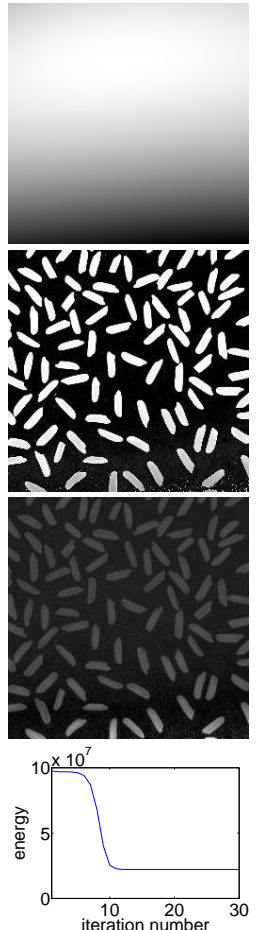
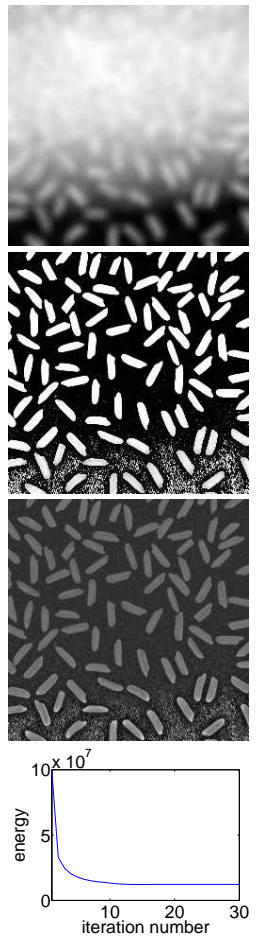
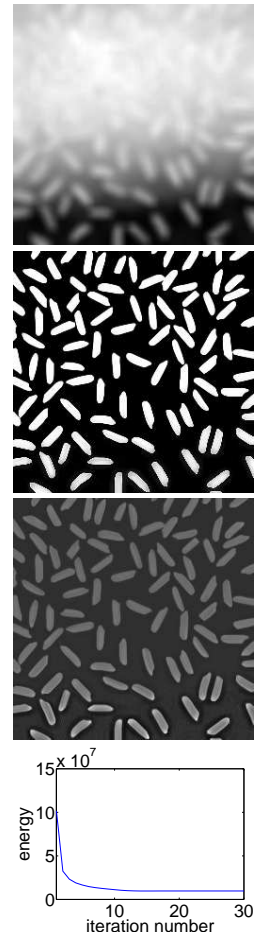

Figure 7: Comparison of NLMD+BCEFCM with FCM, BCFCM, MICO, and BCEFCM on a synthetic image with severe intensity inhomogeneity. The original image, segmentation result of FCM, noise removed by NLMD, and energy curve of FCM are given in the 1st column. Results of BCFCM, MICO, BCEFCM, and NLMD+BCFCM are given in the 2nd to 5th columns, respectively. For each method, the estimated bias field, segmentation result, bias field corrected image, and energy curve are given from top to bottom. 
From results on the second image as shown in Fig. 8, it can be seen that FCM classified a large amount of background pixels to desired segmentation objects due to the negative influence of noise. Similarly, some pixels in the background are considered as parts of segmentation objects by MICO and BCEFCM. Whereas BCFCM classified parts of desired objects into background. It is obvious that NLMD+BCEFCM achieved the best segmentation result and the objective function of BCEFCM converged is less than 10 iterations which is faster than MICO and close to the other methods.
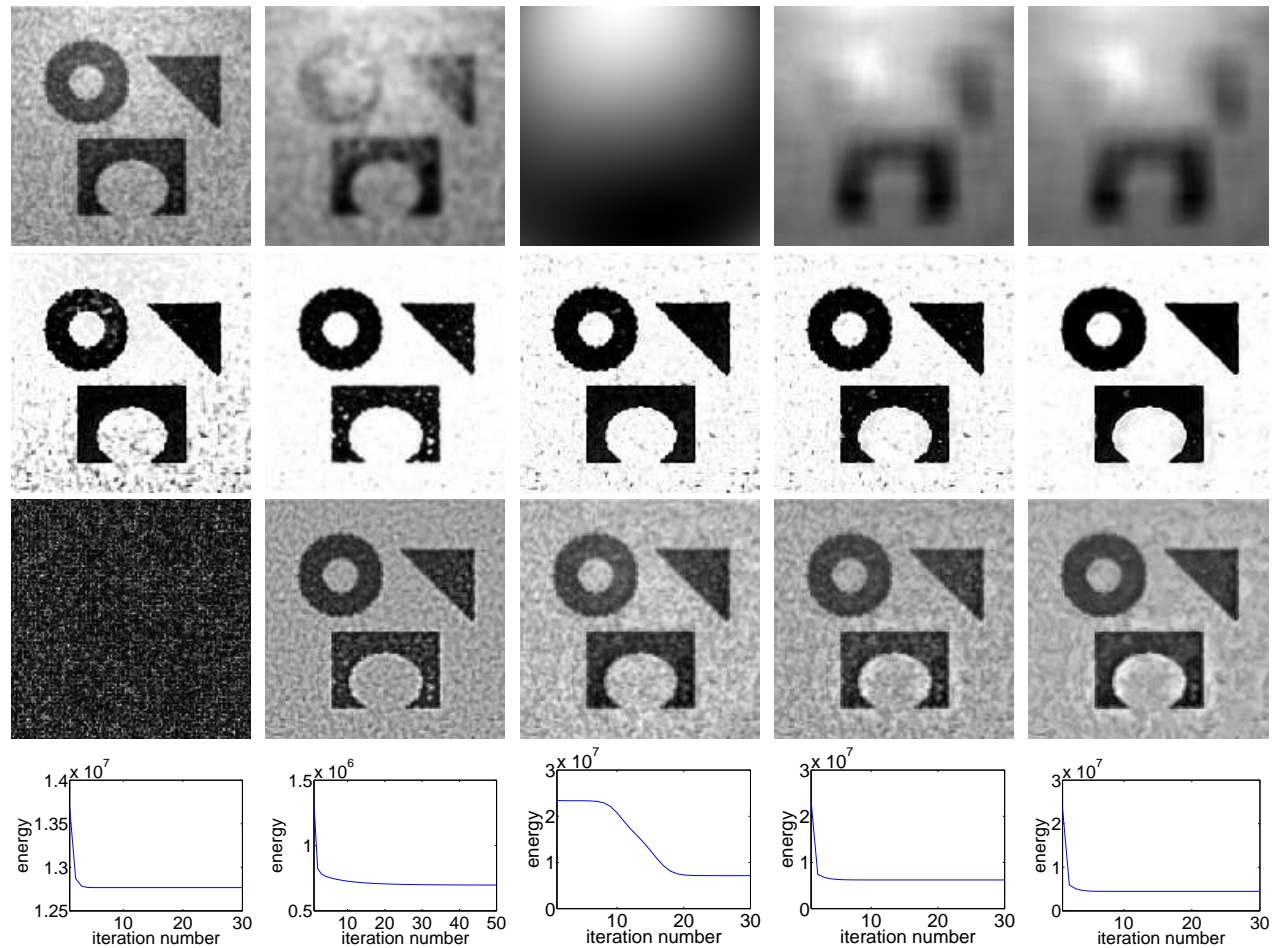

Figure 8: Comparison of NLMD+BCEFCM with FCM, BCFCM, MICO, and BCEFCM on a synthetic image with both severe noise and intensity inhomogeneity. The original image, segmentation result of FCM, noise removed by NLMD, and energy curve of FCM are given in the 1st column. Results of BCFCM, MICO, BCEFCM, and NLMD+BCFCM are given in the 2nd to 5th columns, respectively. For each method, the estimated bias field, segmentation result, bias field corrected image, and energy curve are given from top to bottom. 
From Fig. (9) and Fig. (10) which are results of the proposed framework and state-of-the-art methods on the third image and the fourth image, respectively, we can see that all the other methods considered parts of background as the vessels and the corrected images are still such a mess. However, the proposed NLMD+BCEFCM method extracted the vessels exactly with the objective functions converged in less than 10 iterations, which is faster than BCFCM and MICO and close to FCM.
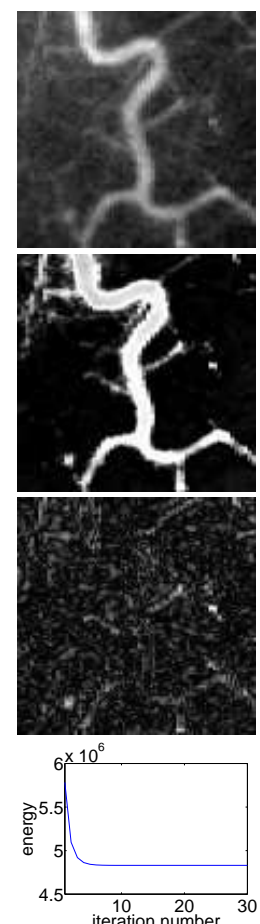
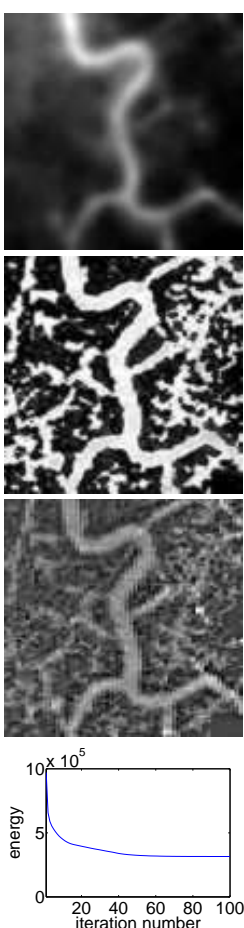
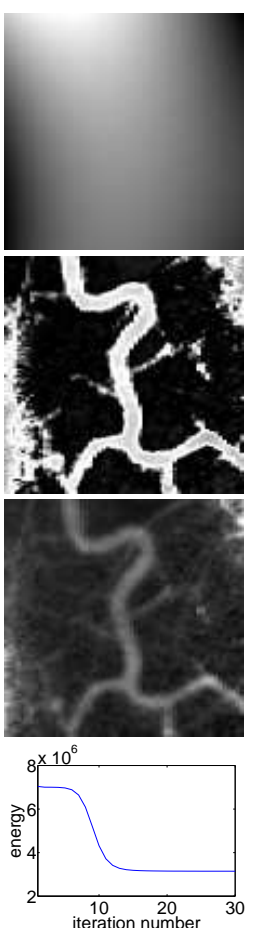
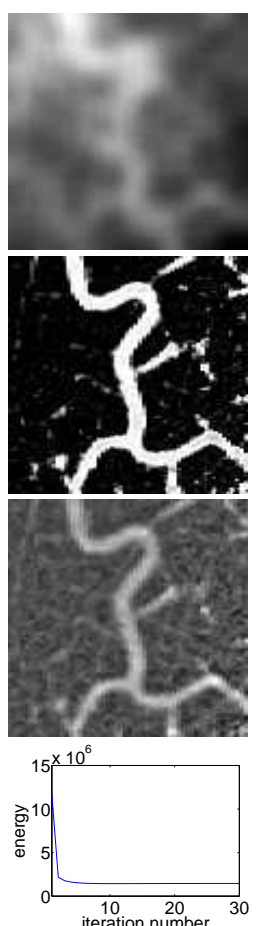
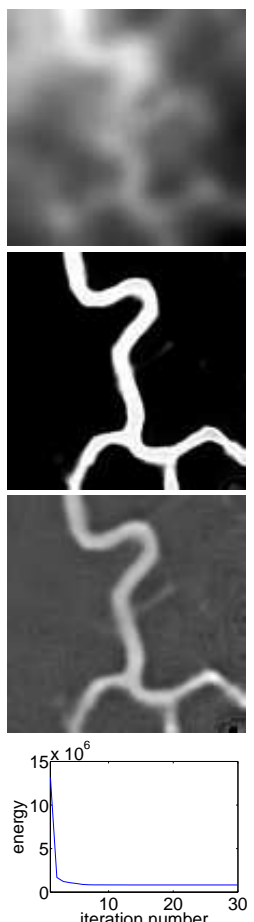

Figure 9: Comparison of NLMD+BCEFCM with FCM, BCFCM, MICO, and BCEFCM on an X-ray image of vessel. The original image, segmentation result of FCM, noise removed by NLMD, and energy curve of FCM are given in the 1st column. Results of BCFCM, MICO, BCEFCM, and NLMD+BCFCM are given in the 2nd to 5th columns, respectively. For each method, the estimated bias field, segmentation result, bias field corrected image, and energy curve are given from top to bottom. 

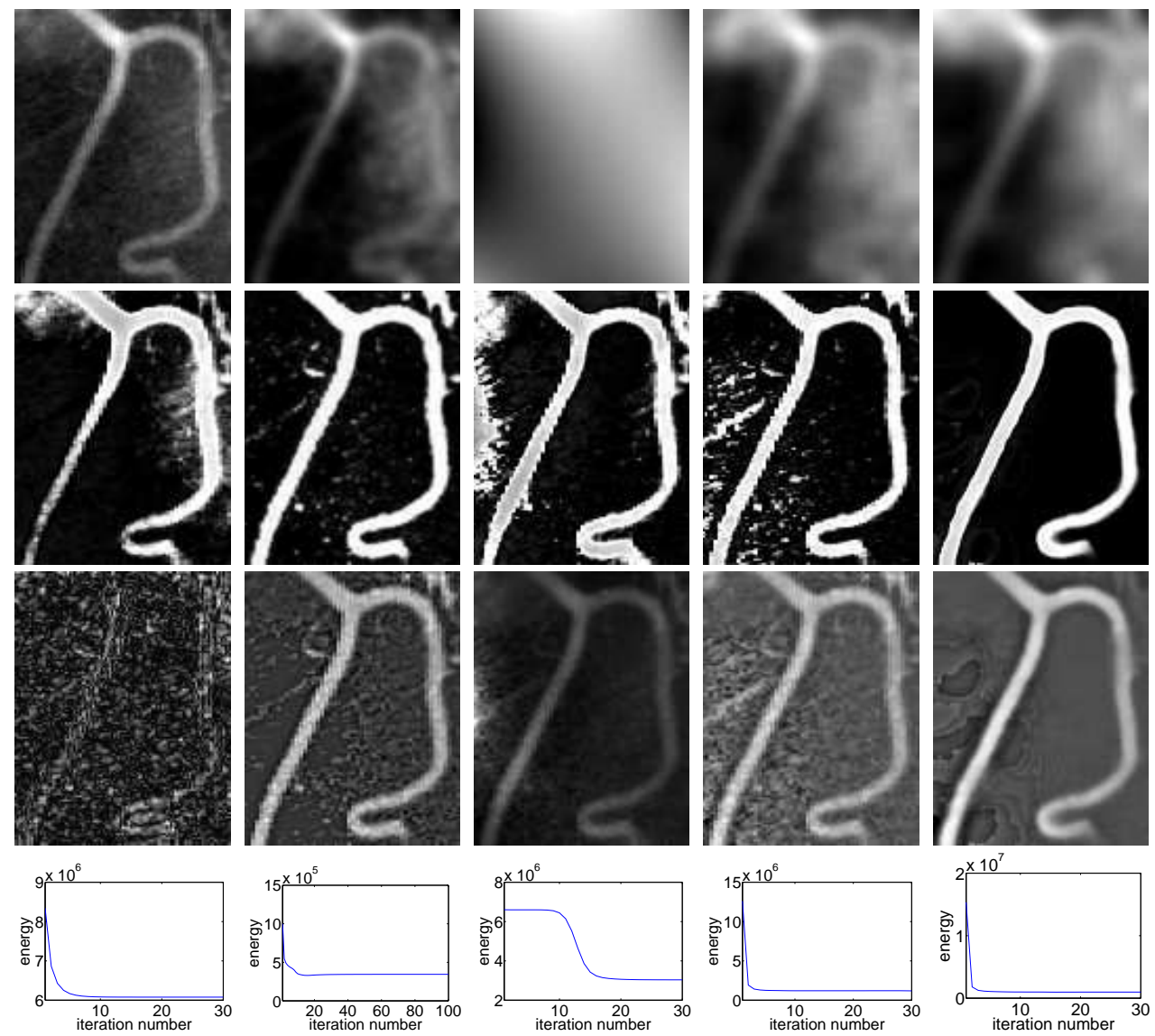

Figure 10: Comparison of NLMD+BCEFCM with FCM, BCFCM, MICO, and BCEFCM on another X-ray image of vessel. The original image, segmentation result of FCM, noise removed by NLMD, and energy curve of FCM are given in the 1st column. Results of BCFCM, MICO, BCEFCM, and NLMD+BCFCM are given in the 2nd to 5th columns, respectively. For each method, the estimated bias field, segmentation result, bias field corrected image, and energy curve are given from top to bottom. 
From Fig. (11), it can be seen that a large mount of WM are considered as GM by FCM. It is obvious that segmentation results of BCFCM are boundary-vague at bottom of the image. And segmentation results of MICO and BCEFCM are affected by the noise existing in the image. Obviously, NLMD+BCEFCM achieved the best segmentation in less than 10 iterations which is the fastest convergence.
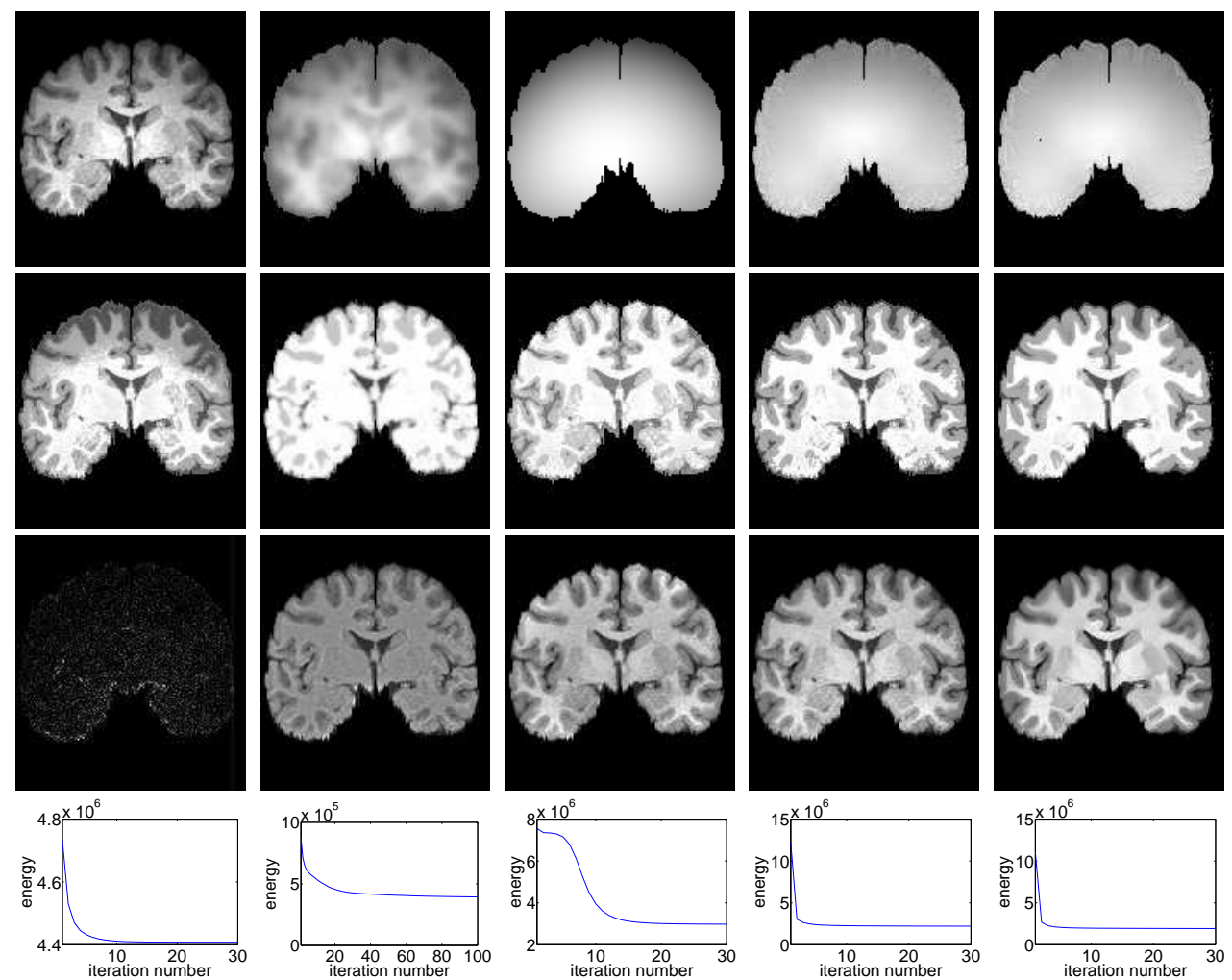

Figure 11: Comparison of NLMD+BCEFCM with FCM, BCFCM, MICO, and BCEFCM on an MR image of brain. The original image, segmentation result of FCM, noise removed by NLMD, and energy curve of FCM are given in the 1st column. Results of BCFCM, MICO, BCEFCM, and NLMD+BCFCM are given in the 2nd to 5th columns, respectively. For each method, the estimated bias field, segmentation result, bias field corrected image, and energy curve are given from top to bottom.

As shown in Fig. 12, Fig. 13, and Fig. 14, the proposed method is pixel-based which leads to small background objects ignored by the ground truth are recognized. But the proposed method is still much more robust than others: 1) FCM is ineffective in segmenting the second image from BSD. 2) Although bias fields estimated by MICO are much more smooth than other methods, there are obvious errors in segmentation results of MICO as shown in Fig. 12 and Fig. 13, 3) For the first and third images from BSD, BCFCM is not able to offer satisfactory segmentation results. 


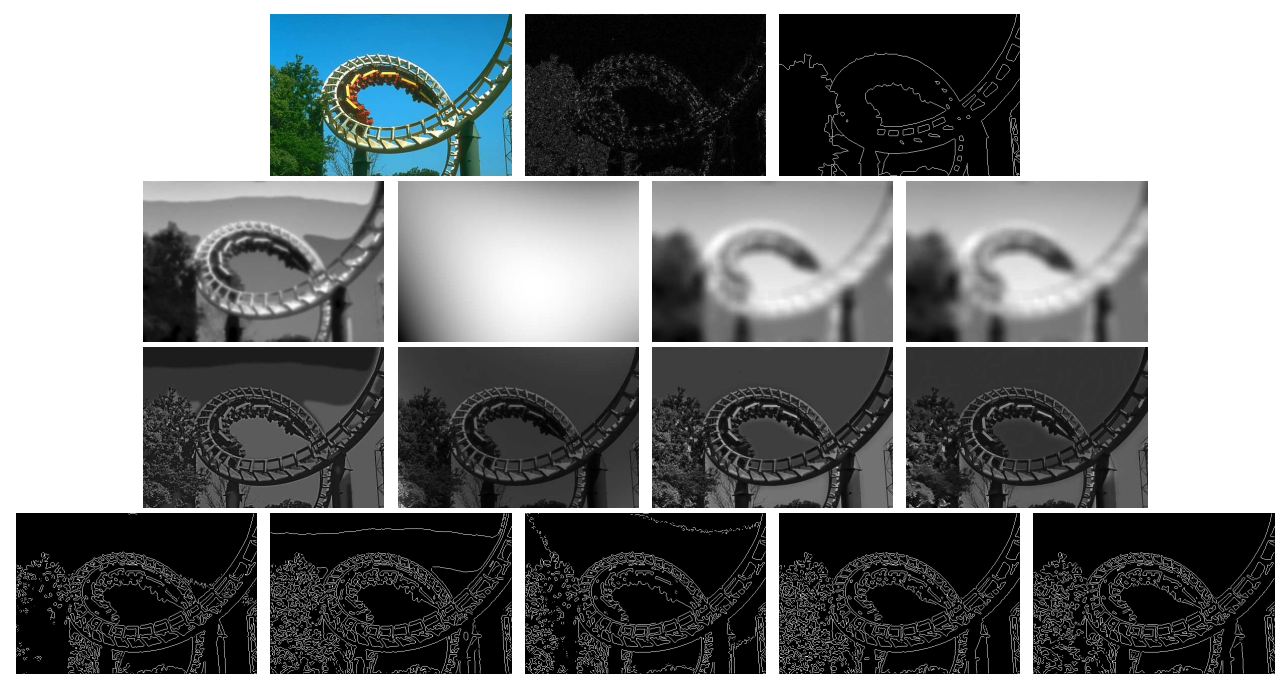

Figure 12: Comparison of NLMD+BCEFCM with FCM, BCFCM, MICO, and BCEFCM on a natural image (235098) from BSD database. The original image, noise removed by NLMD, and one of the ground truths are given in the 1st row. Estimated bias fields and bias corrected results of BCFCM, MICO, BCEFCM, and NLMD+BCEFCM are given in the 2nd and 3rd rows from left to right, respectively. Boundaries detected using matlab edge function with Laplacian of Gaussian method from segmentation results of FCM, BCFCM, MICO, BCEFCM, and NLMD+BCEFCM are given in the 4th row from left to right, respectively.

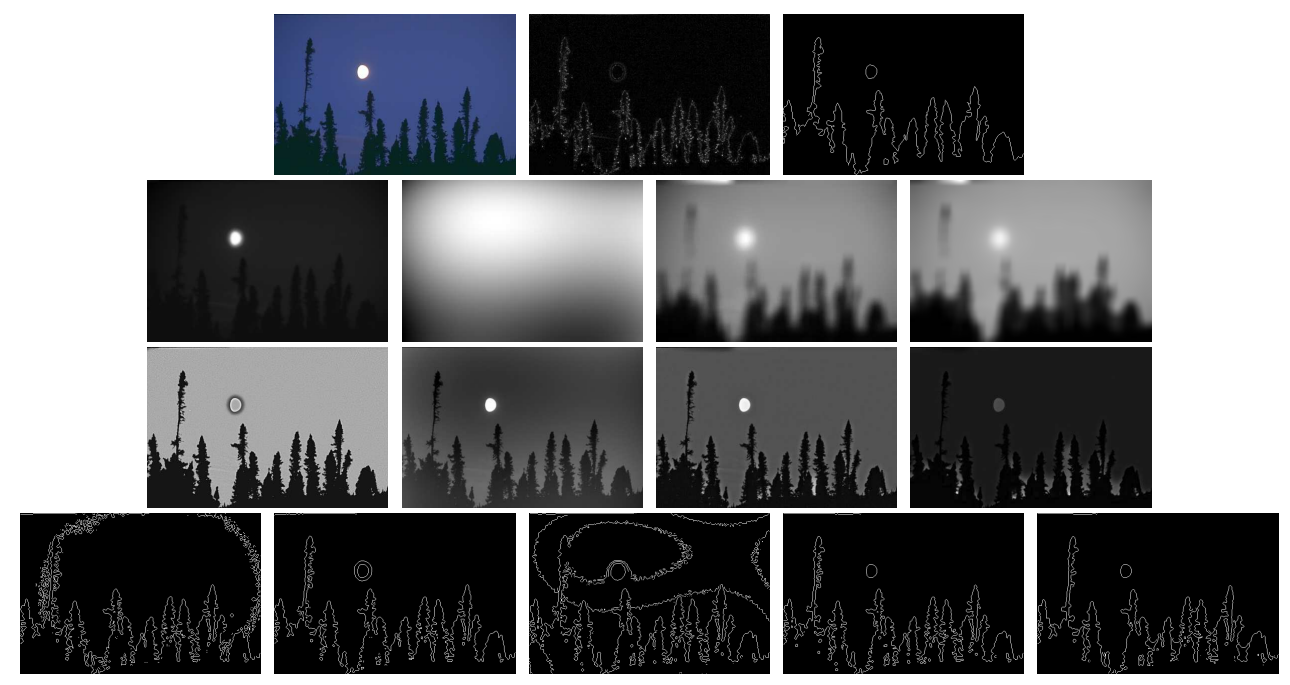

Figure 13: Comparison of NLMD+BCEFCM with FCM, BCFCM, MICO, and BCEFCM on a natural image (238011) from BSD database. The original image, noise removed by NLMD, and one of the ground truths are given in the 1st row. Estimated bias fields and bias corrected results of BCFCM, MICO, BCEFCM, and NLMD+BCEFCM are given in the 2nd and 3rd rows from left to right, respectively. Boundaries detected using matlab edge function with Laplacian of Gaussian method from segmentation results of FCM, BCFCM, MICO, BCEFCM, and NLMD+BCEFCM are given in the 4th row from left to right, respectively. 


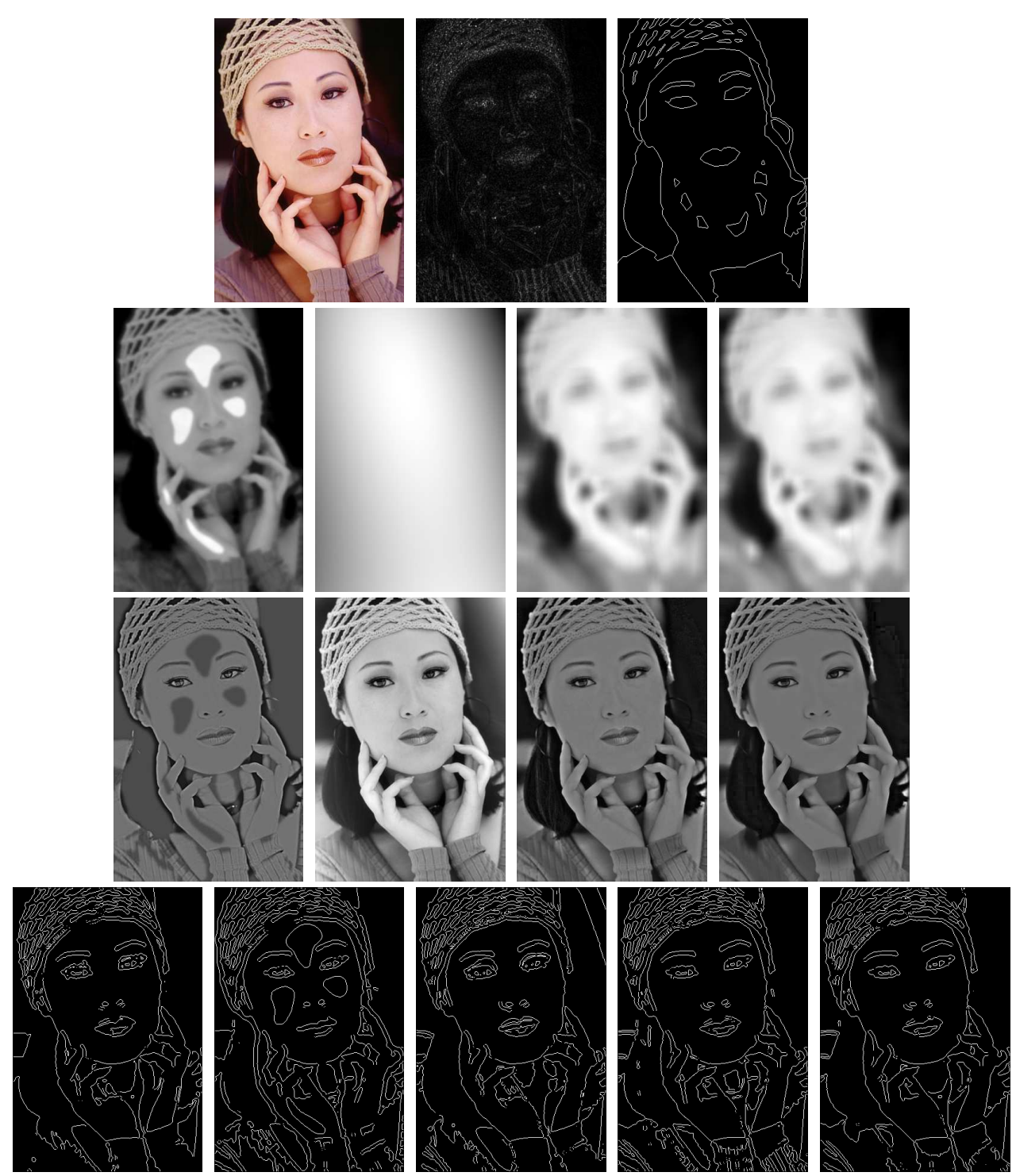

Figure 14: Comparison of NLMD+BCEFCM with FCM, BCFCM, MICO, and BCEFCM on a natural image (302003) from BSD database. The original image, noise removed by NLMD, and one of the ground truths are given in the 1st row. Estimated bias fields and bias corrected results of BCFCM, MICO, BCEFCM, and NLMD+BCEFCM are given in the 2nd and 3rd rows from left to right, respectively. Boundaries detected using matlab edge function with Laplacian of Gaussian method from segmentation results of FCM, BCFCM, MICO, BCEFCM, and NLMD+BCEFCM are given in the 4th row from left to right, respectively. 
The iteration number and CPU times of FCM, BCFCM, MICO, BCEFCM, and NLMD+BCEFCM on segmenting the images shown in Fig. (7), Fig. (8), Fig. (9), Fig. (10), and Fig. (11) are listed in Table 1 It is obvious that the proposed framework is just a little slower than FCM but faster than all the others in both convergence speed and run time.

Table 1: Comparison of NLMD+BCEFCM with the-state-of-art methods in terms of iterations and run time (in seconds).

\begin{tabular}{|c|c|c|c|c|c|c|c|c|c|c|}
\hline method & \multicolumn{2}{|c|}{$\begin{array}{ll}\text { Image in } & \text { Fig. } \\
\text { iters } & \text { time }\end{array}$} & \multicolumn{2}{|c|}{$\begin{array}{l}\text { Image in Fig. } 8 \\
\text { iters time }\end{array}$} & \multicolumn{2}{|c|}{$\begin{array}{l}\text { Image in Fig. } 9 \\
\text { iters time }\end{array}$} & \multicolumn{2}{|c|}{$\begin{array}{l}\text { Image in Fig. } 10 \\
\text { iters time }\end{array}$} & \multicolumn{2}{|c|}{$\begin{array}{lc}\text { Image in } & \text { Fig. } 11 \\
\text { iters } & \text { time }\end{array}$} \\
\hline$\overline{\mathrm{FCM}}$ & 6 & 0.0408 & 5 & 0.0190 & 9 & 0.0098 & 12 & 0.0136 & 10 & 0.0279 \\
\hline BCFCM & 23 & 43.6631 & 23 & 16.1020 & 92 & 32.4060 & 46 & 18.3156 & 79 & 65.6465 \\
\hline MICO & 16 & 0.4367 & 21 & 0.1937 & 21 & 0.1387 & 24 & 0.1313 & 27 & 0.2532 \\
\hline BCEFCM & 22 & 0.2713 & 12 & 0.1055 & 27 & 0.0797 & 52 & 0.1785 & 72 & 0.7583 \\
\hline NLMD+BCEFCM & 17 & 0.2003 & 10 & 0.0484 & 13 & 0.0364 & 18 & 0.0535 & 100 & 0.9110 \\
\hline
\end{tabular}

\subsection{Quantitative Evaluation of Segmentation Accuracy}

To evaluate segmentation accuracy of the proposed framework quantitatively, we compare segmentation results of NLMD+BCEFCM with FCM, BCFCM, MICO, and BCEFCM in terms of false positive ratio (FPR), false negative ratio (FNR), and dice similarity coefficient (DSC) on both BrainWeb and IBSR datasets in this subsection.

For image segmentation, a false positive (FP) occurs when a segmentation result indicates that a pixel belongs to a tissue, but it does not in fact. On the contrary, a false negative (FN) occurs when the segmentation result indicates that a pixel does not belong to a tissue, but it does in fact. Let NFP and NFN be the number of FP and FN and $A$ be the ground truth, FPR and FNR can then be defined by

$$
\mathrm{FPR}=\frac{\mathrm{NFP}}{|I|-|A|} \times 100 \%
$$

and

$$
\mathrm{FNR}=\frac{\mathrm{NFN}}{|A|} \times 100 \%
$$

respectively, where $|*|$ denotes the size of region $*$. Obviously, values of FPR and FNR both range from 0 and 1 with a smaller value indicating a better match between the segmentation result and ground truth.

To compare segmentation accuracy of the proposed framework with state-of-the-art methods more objectively and precisely, we also evaluate segmentation results quantitatively with the metric of dice similarity coefficient in this subsection. It is well known that DSC is defined as twice of the quotient between intersection size of a pairwise variables and sum of their sizes where the variables are a segmentation result and the ground truth for image segmentation. Let $B$ be the segmentation, definition of DSC can therefore be written as

$$
D S C=\frac{2|A \cap B|}{|A|+|B|}
$$


where $\cap$ is the intersection operator. It is obvious that values of $D S C$ are in the interval of $[0,1]$ with a higher value indicating a better match between the segmentation result $B$ and the ground truth $A$.

We first evaluate segmentation accuracy of the proposed framework quantitatively on T1 simulated MR images downloaded from http://brainweb.bic.mni.mcgill.ca/brainweb/. We first downloaded 9 cases of MR images with three different levels of noise (none, 5\%, and $9 \%$ to the brightest tissue) and three levels of intensity inhomogeneity (none, 20\%, and $40 \% \mathrm{RF}$ ) from the website. Resolutions of the images are $181 \times 217 \times 181$ with $1 \mathrm{~mm}$ in-plane pixel size and $1 \mathrm{~mm}$ slice thickness. Interested readers are referred to the website of BrainWeb and reference [43] for more details about the dataset. Note that intensity inhomogeneities in the images are linear and they are therefore easily handled by segmentation methods. To examine segmentation methods in a much more difficult situation, we added three levels of non-linear intensity inhomogeneities to the simulated image by multiplying them with the original image downloaded from BrainWeb to obtain three images with different intensity inhomogeneities. We then added noise of three different levels to these images and therefore obtained another 9 cases of MR images. We applied the proposed framework to segment these image cases with $\lambda_{1}=1.0, \lambda_{2}=1.0$, and $\lambda_{3}=1.5$. Cluster centroids $\left\{c_{i}\right\}_{i=1}^{3}$ are initialized as equally spaced values in the range of intensities for each image case. We first show the 90-th image slice of each of the above mentioned 18 cases in Fig. 15. It can be seen that the images are corrupted by different levels of noise and intensity inhomogeneities. We then show segmentation results of NLMD+BCEFCM and the state-of-the-art methods on six slices of the image case with $9 \%$ noise and $40 \%$ linear intensity inhomogeneity and six slices of the image case with the most severe noise and non-linear intensity inhomogeneity in Fig. 16 and Fig. 17 respectively. It can be obviously seen that 1) segmentation results of FCM are the most easily affected by both noise and intensity inhomogeneity, 2) BCFCM considered parts of GM as WM, 3) BCFCM, MICO, and BCEFCM are sensitive to noise, and 4) NLMD+BCEFCM is not sensitive either to noise or to intensity inhomogeneity with its segmentation results match with the ground truth best. We finally calculate FPR, FNR, and DSC values for WM, GM, and CSF obtained from the proposed framework, FCM, BCFCM, MICO, and BCEFCM on BrainWeb dataset, respectively and show the results with boxplots in Fig. 18. Note that a better match between a segmentation result and the ground truth will result in lower values not only for FPR but also for FNR. Obviously, the boxes of FPR and FNR values shown in Fig. 18 for NLMD+BCEFCM are relatively shorter and lower than the others and there are no outliers, which exhibits desirable stability of the proposed framework in segmenting images from BrainWeb. Furthermore, although there are outliers in DSC values for WM, GM, and CSF, the boxes of NLMD+BCEFCM are relatively shorter and higher than all the others and the outliers are obviously greater than outliers in the other methods. Therefore, segmentation results of NLMD+BCEFCM matched with the ground truth better than segmentation results of the state-of-the-art methods on BrainWeb images. 


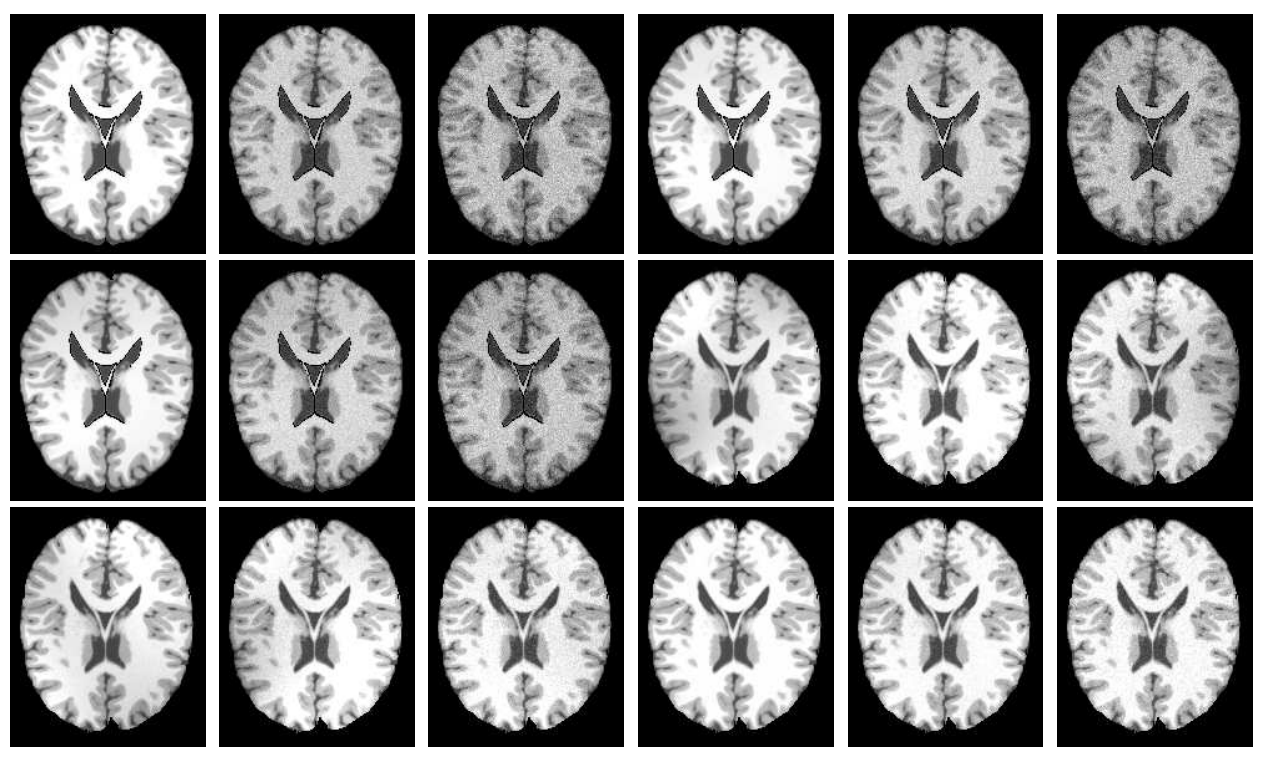

Figure 15: Images from BrainWeb: each from one case. 


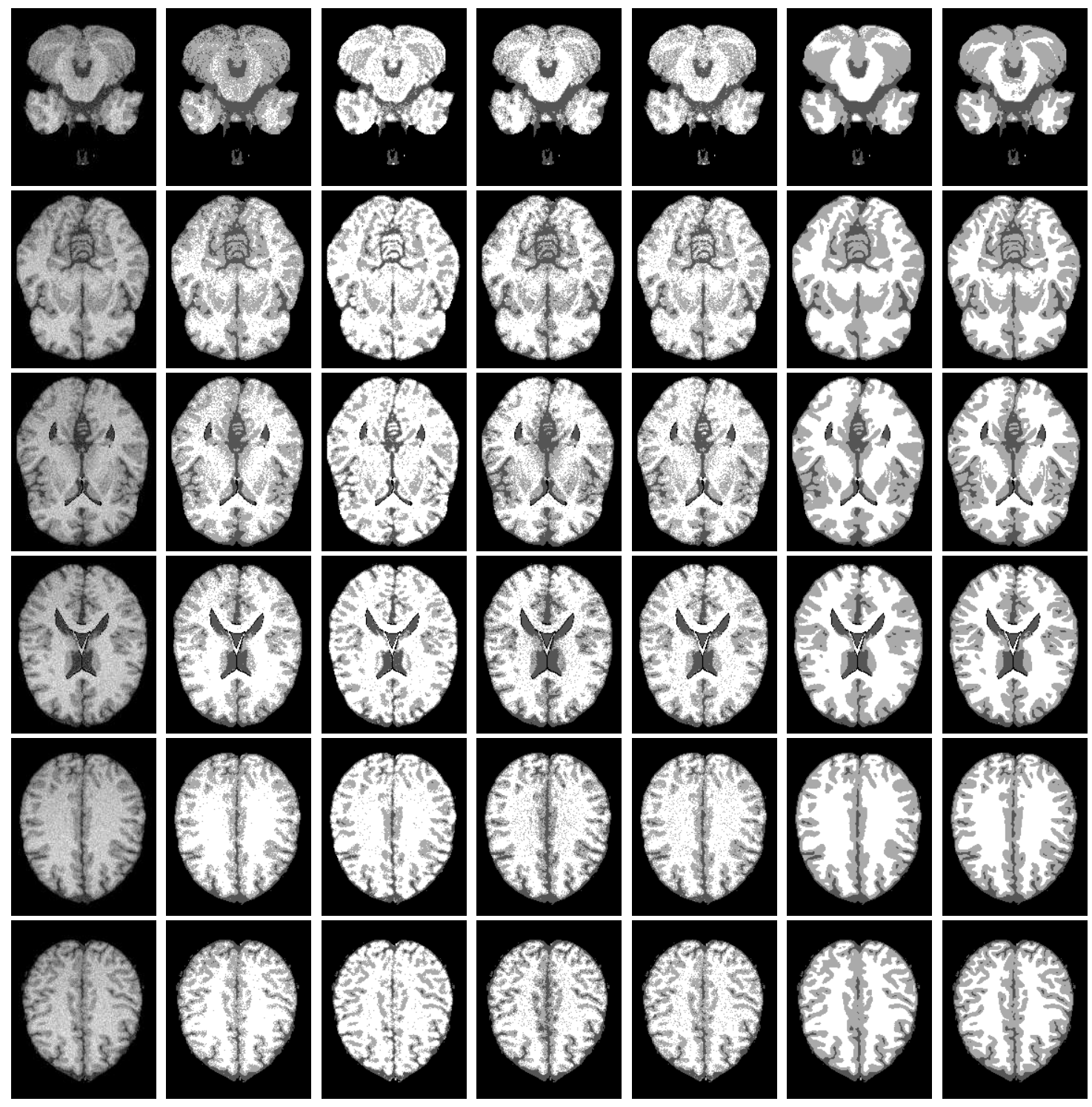

Figure 16: Comparison of segmentation results of NLMD+BCEFCM with state-of-the-art methods on six slices of the image case from BrainWeb with $9 \%$ noise to the brightest tissue and $40 \%$ linear intensity inhomogeneity. From left to right: original images, segmentation results of FCM, BCFCM, MICO, BCEFCM, NLMD+BCEFCM, and the ground truth. 


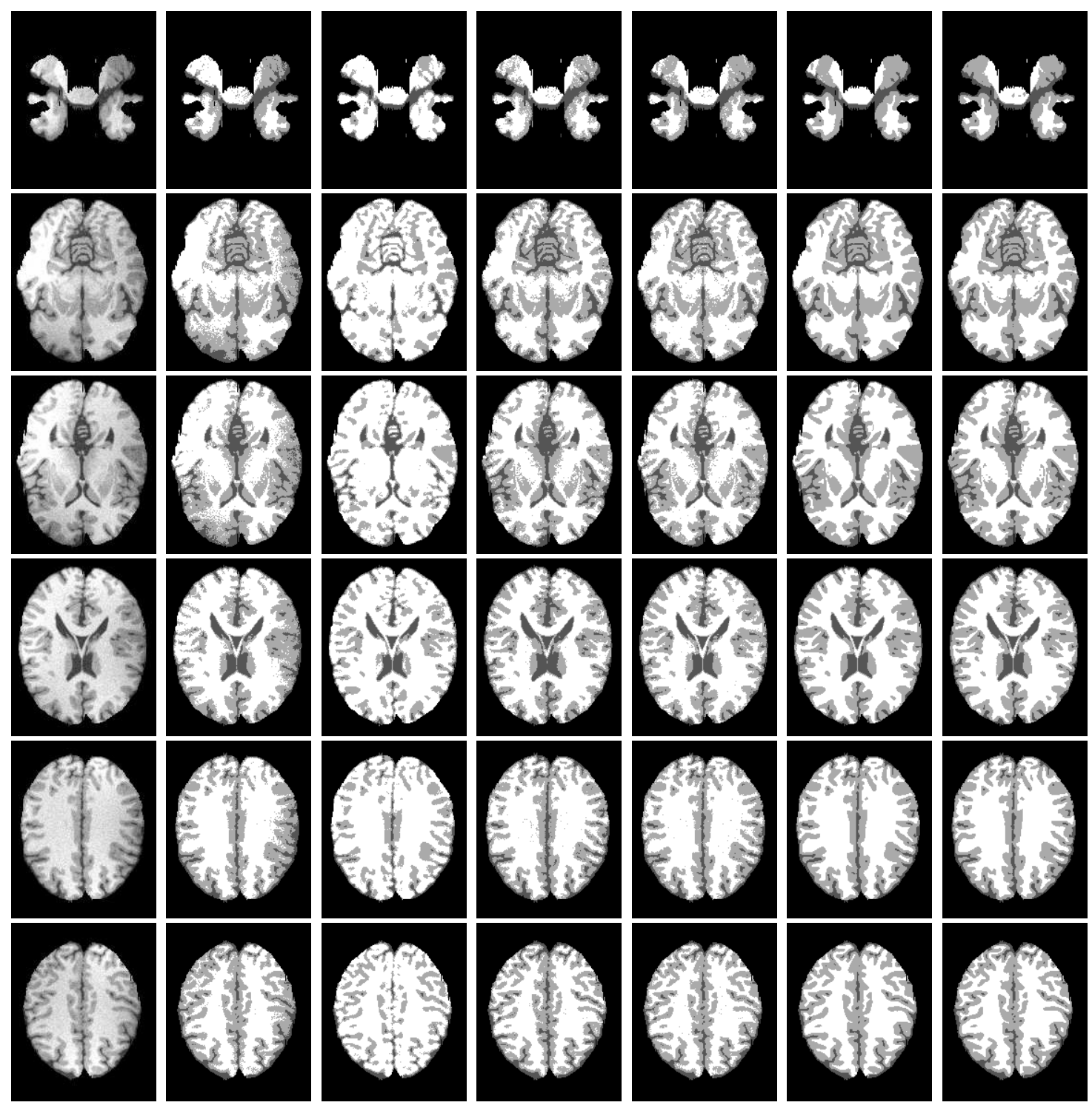

Figure 17: Comparison of segmentation results of NLMD+BCEFCM with state-of-the-art methods on six slices of one image case from BrainWeb with the most severe noise and non-linear intensity inhomogeneity. From left to right: original images, segmentation results of FCM, BCFCM, MICO, BCEFCM, NLMD+BCEFCM, and the ground truth. 

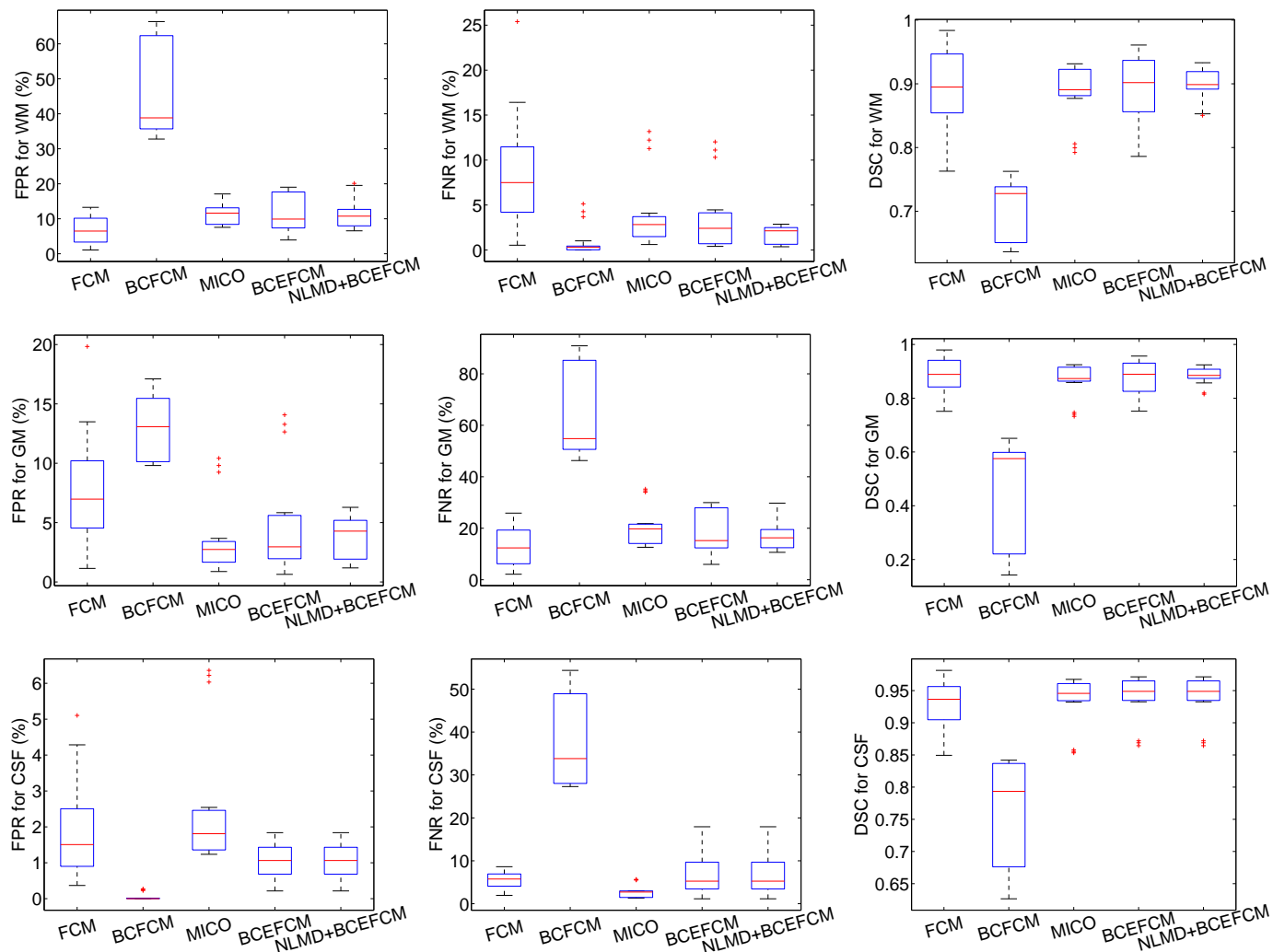

Figure 18: Quantitative comparison of the proposed framework NLMD+BCEFCM with FCM, BCFCM, MICO, and BCEFCM on BrainWeb dataset. 
We then evaluate segmentation accuracy of the proposed framework quantitatively on real MR images downloaded from the Internet Brain Segmentation Repository (IBSR) which is a World Wide Web resource providing access to MR brain images and segmentation results contributed and utilized by researchers from all over the world. The IBSR is supported by the National Institute of Neurological Disorders and Stroke at the NIH part of a grant that funds research in MR brain segmentation by researchers at Boston University, Draper Laboratory, Northeastern University, Massachusetts Institute of Technology, and Massachusetts General Hospital / Harvard Medical School. Its purpose is to encourage the development and evaluation of segmentation methods by providing raw test and image data, human expert segmentation results, and methods for comparing segmentation results. The IBSR dataset contains 18 cases of T1-weighted brain MR images together with skull-removed masks and manually-guided expert segmentation results (groundtruth). Resolutions of the images are all $256 \times 128 \times 256$. Interested readers are referred to the website https://www.nitrc.org/projects/ibsr and reference [4] for more details about the dataset. We applied the proposed framework to segment these image cases with $\lambda_{1}=2.0, \lambda_{2}=0.5$, and $\lambda_{3}=2.0$. Cluster centroids $\left\{c_{i}\right\}_{i=1}^{3}$ are initialized as equally spaced values in the range of intensities for each image case. We first show the 128-th image slice of each of the above mentioned 18 cases and the corresponding ground truth in Fig. 19. We then compare segmentation results of NLMD+BCEFCM with the state-of-the-art methods on six slices of the image case IBSR_04 and show the results in Fig. 20. It can be seen that 1) parts of GM are considered as WM by BCFCM, 2) there are too much CSF in segmentation results of MICO, 3) the amount of CSF and WM are both obviously greater than the ground truth in segmentation results of FCM, and 4) segmentation results of BCEFCM and NLMD+BCEFCM match with the ground truth best and the latter are not sensitive to noise. We finally calculate FPR, FNR, and DSC values for WM, GM, and CSF obtained from the proposed framework, FCM, BCFCM, MICO, and BCEFCM on the dataset, respectively and show the results with boxplots in Fig. 21, It can be seen from Fig. 21 that FPR and FNR values of NLMD+BCEFCM balanced better (either of them are too great or small) which indicates that the segmentation result of NLMD+BCEFCM match with the ground truth better. Although there are one outlier for WM and two outliers for GM, the boxes of NLMD+BCEFCM are relatively shorter and higher than all the others. Above all, segmentation results of NLMD+BCEFCM matched with the ground truth better than segmentation results of the state-of-the-art methods on IBSR images. 


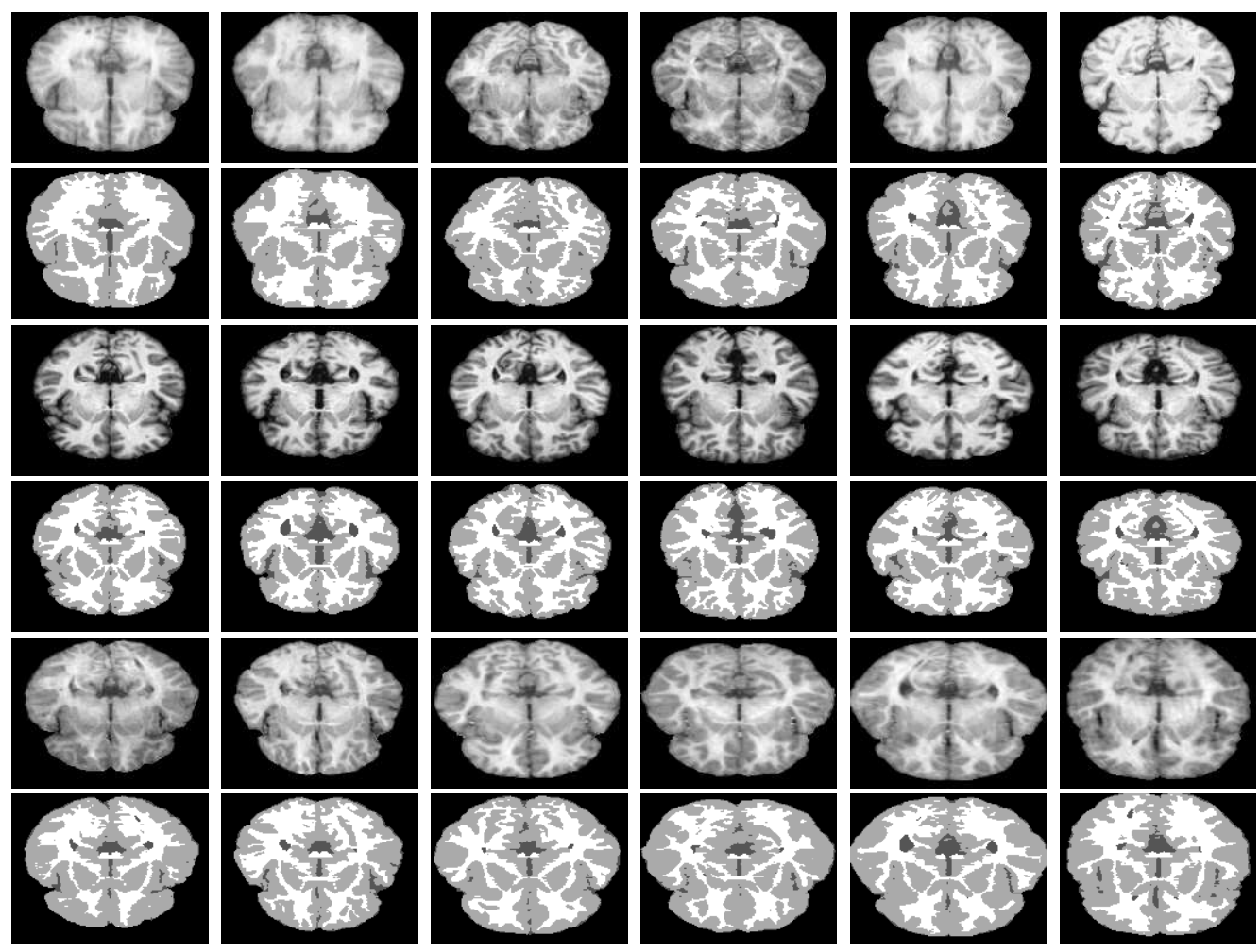

Figure 19: Images (in odd rows) and the ground truth (in even rows) from IBSR: each from one case. 


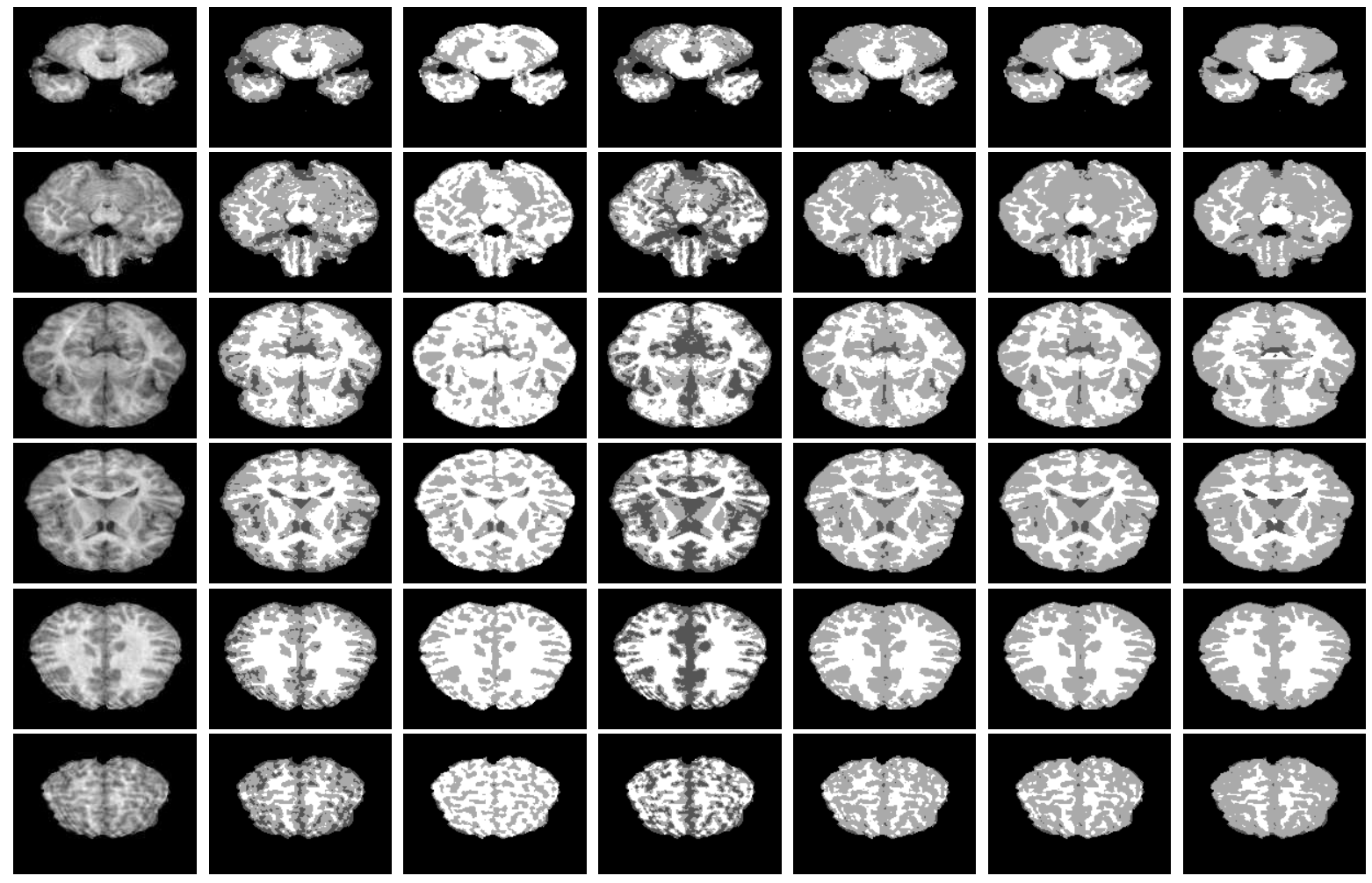

Figure 20: Comparison of segmentation results of NLMD+BCEFCM with state-of-the-art methods on six slices from case IBSR_04 in six rows. For each slice, the original image, segmentation result of FCM, BCFCM, MICO, BCEFCM, NLMD+BCEFCM, and the ground truth are given from left to right, respectively. 

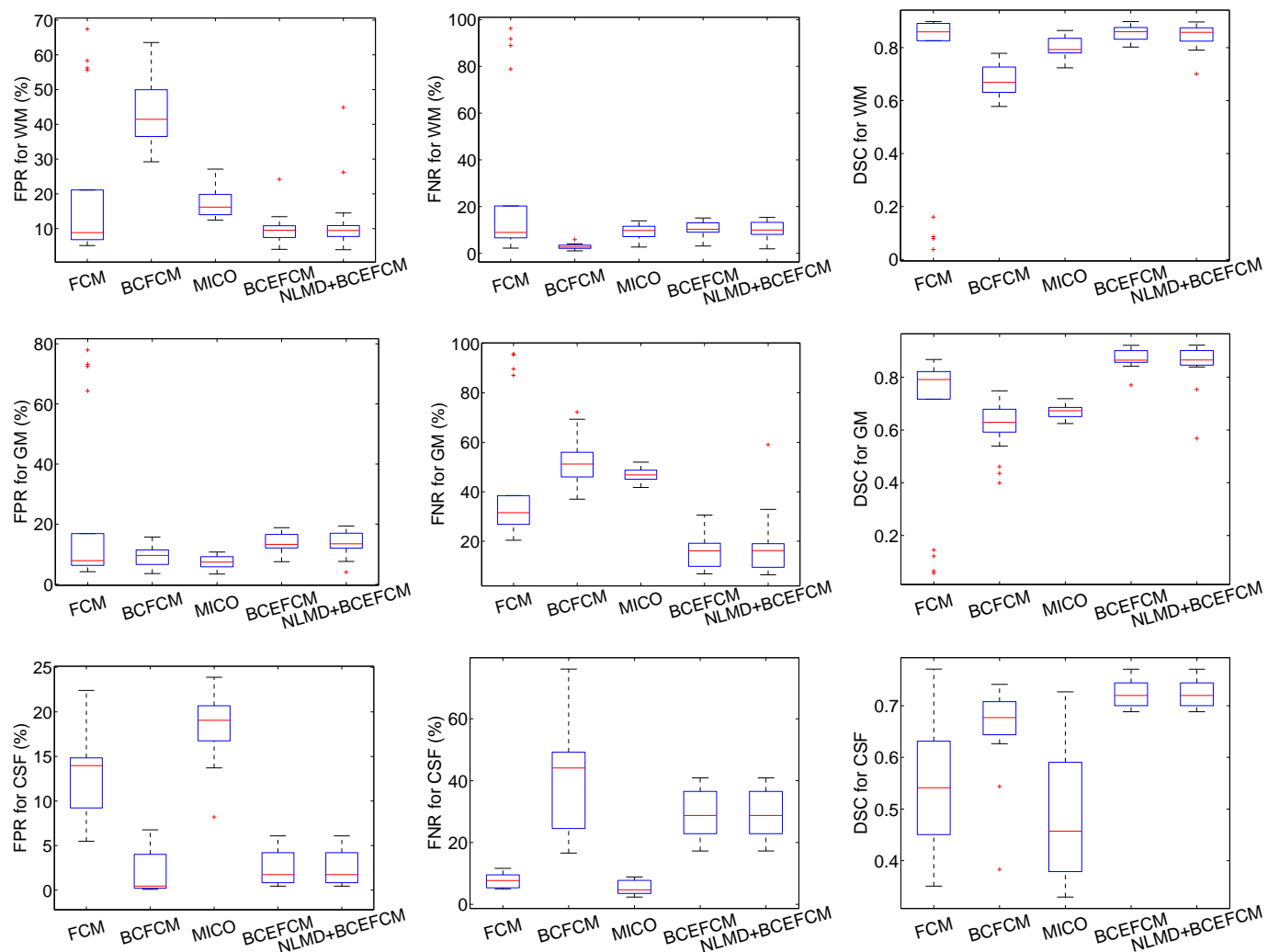

Figure 21: Quantitative comparison of the proposed framework NLMD+BCEFCM with FCM, BCFCM, MICO, and BCEFCM on IBSR dataset. 


\section{Discussions}

\subsection{Robustness of BCEFCM to Initialization}

It is worth pointing out that the proposed BCEFCM is robust to initialization. To demonstrate this visually, we applied it to a brain image from BrainWeb with four different initializations of the membership functions $u_{1}, u_{2}, \ldots, u_{N}$ and the intensity constants of different tissues $c_{1}, c_{2}, \ldots, c_{N}$. We show the original image in the upper left corner of Fig. 22, The initialization can be visualized as an image defined by $J(\mathbf{x})=\sum_{i=1}^{N} c_{i} u_{i}(\mathbf{x})$ as shown in the first column of Fig. 22 from the second row to the last row. In particular, the first initialization shown in the first column and the second row of Fig. 22 is obtained by initializing the membership functions and intensity constants of different tissues as random numbers. For these four different initializations, the estimated bias fields, the segmentation results, and the corrected images are the same up to a negligible difference as shown in the second to fourth columns of Fig. 22, We also plot the energy $F_{b c e f c m}$ computed at each iteration for 50 iterations and show the result in the upper right conner of Fig. 22 It is obvious that the energy $F_{b c e f c m}$ decreases to the same minimum value from the four different initializations in less than 40 iterations. Above all, the proposed BCEFCM is robust to initialization.

\subsection{Impact of Weighting Coefficients $\lambda_{i}$}

Let $\gamma_{i}(\mathbf{x})$ be $\left\|N L(I)(\mathbf{x})-b(\mathbf{x}) c_{i}\right\|^{2}$ and $n=\frac{1}{q-1}$, then Eq.(33) can be rewritten as

$$
\begin{aligned}
\breve{u}_{i}(\mathbf{x}) & =\frac{1}{\sum_{j=1}^{N}\left(\frac{\lambda_{i} \gamma_{i}(\mathbf{x})}{\lambda_{j} \gamma_{j}(\mathbf{x})}\right)^{n}} \\
& =\frac{1}{\sum_{j=1}^{i-1}\left(\frac{\lambda_{i} \gamma_{i}(\mathbf{x})}{\lambda_{j} \gamma_{j}(\mathbf{x})}\right)^{n}+\sum_{j=i+1}^{N}\left(\frac{\lambda_{i} \gamma_{i}(\mathbf{x})}{\lambda_{j} \gamma_{j}(\mathbf{x})}\right)^{n}+1} .
\end{aligned}
$$

If $\Delta \lambda_{i}>0$, we substitute $\lambda_{i}^{\prime}=\lambda_{i}+\Delta \lambda_{i}$ into the above equation and obtain

$$
\breve{u}_{i}^{\prime}(\mathbf{x})=\frac{1}{\sum_{j=1}^{i-1}\left(\frac{\left(\lambda_{i}+\Delta \lambda_{i}\right) \gamma_{i}(\mathbf{x})}{\lambda_{j} \gamma_{j}(\mathbf{x})}\right)^{n}+\sum_{j=i+1}^{N}\left(\frac{\left(\lambda_{i}+\Delta \lambda_{i}\right) \gamma_{i}(\mathbf{x})}{\lambda_{j} \gamma_{j}(\mathbf{x})}\right)^{n}+1} .
$$

Thus, difference between $\breve{u}_{i}^{\prime}(\mathbf{x})$ and $\breve{u}_{i}(\mathbf{x})$ can be written as

$$
\begin{aligned}
\Delta \breve{u}_{i}(\mathbf{x}) & =\breve{u}_{i}^{\prime}(\mathbf{x})-\breve{u}_{i}(\mathbf{x}) \\
& =\frac{\sum_{j=1}^{i-1}\left(\left(\frac{\lambda_{i} \gamma_{i}(\mathbf{x})}{\lambda_{j} \gamma_{j}(\mathbf{x})}\right)^{n}-\left(\frac{\left(\lambda_{i}+\Delta \lambda_{i}\right) \gamma_{i}(\mathbf{x})}{\lambda_{j} \gamma_{j}(\mathbf{x})}\right)^{n}\right)+\sum_{j=i+1}^{N}\left(\left(\frac{\lambda_{i} \gamma_{i}(\mathbf{x})}{\lambda_{j} \gamma_{j}(\mathbf{x})}\right)^{n}-\left(\frac{\left(\lambda_{i}+\Delta \lambda_{i}\right) \gamma_{i}(\mathbf{x})}{\lambda_{j} \gamma_{j}(\mathbf{x})}\right)^{n}\right)}{\left(\sum_{j=1}^{N}\left(\frac{\lambda_{i} \gamma_{i}(\mathbf{x})}{\lambda_{j} \gamma_{j}(\mathbf{x})}\right)^{n}\right)\left(\sum_{j=1}^{N}\left(\frac{\left(\lambda_{i}+\Delta \lambda_{i}\right) \gamma_{i}(\mathbf{x})}{\left(\lambda_{j}+\Delta \lambda_{j}\right) \gamma_{j}(\mathbf{x})}\right)^{n}\right)}
\end{aligned}
$$

45 It is obvious that $\frac{\left(\lambda_{i}+\Delta \lambda_{i}\right) \gamma_{i}(\mathbf{x})}{\lambda_{j} \gamma_{j}(\mathbf{x})}>\frac{\lambda_{i} \gamma_{i}(\mathbf{x})}{\lambda_{j} \gamma_{j}(\mathbf{x})}>0$ due to $\gamma_{i}(\mathbf{x})>0, \gamma_{j}(\mathbf{x})>0, \lambda_{i}>0, \lambda_{j}>0$, and $\Delta \lambda_{i}>0$ for $i=1,2, \ldots, N, j=1,2, \ldots, N$, and $i \neq j$. It is well known that the power function $x^{n}$ is positive and is 


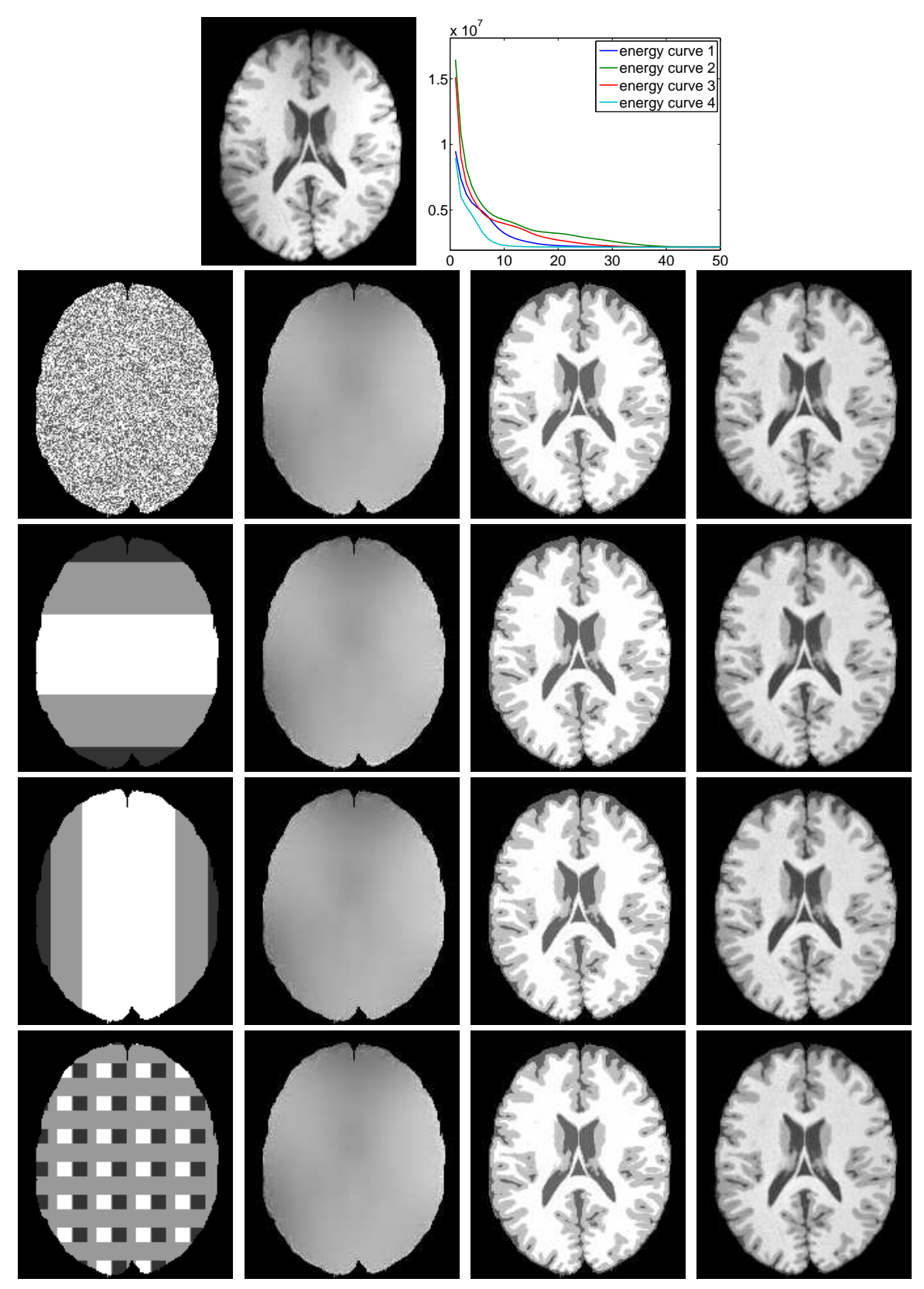

Figure 22: Demonstration of robustness of BCEFCM to initialization. The original image and energy decreasing curves are given in the 1st row. Four initializations and corresponding results of BCEFCM are given in four rows. For each row, the initialization, estimated bias field, segmentation result, and corrected image are given in different columns from left to right, respectively.

477 monotonically increasing in the interval of $(0, \infty)$ if $n>0$. Thus, $\left(\frac{\left(\lambda_{i}+\Delta \lambda_{i}\right) \gamma_{i}(\mathbf{x})}{\lambda_{j} \gamma_{j}(\mathbf{x})}\right)^{n}>\left(\frac{\lambda_{i} \gamma_{i}(\mathbf{x})}{\lambda_{j} \gamma_{j}(\mathbf{x})}\right)^{n}$. Therefore, 478 for the case $q>1, \Delta \breve{u}_{i}(\mathbf{x})<0$ if $\Delta \lambda_{i}>0$. For the case $q=1$, it can be seen obviously from Eq. (34) that the 479 increase of $\lambda_{i}$ will decrease the possibility of $\operatorname{argmin}_{i}\left(\lambda_{i} \gamma_{i}\right)$ being $i$. That is to say, $u_{i}(\mathbf{x})$ can been viewed as a monotonic decreasing function of $\lambda_{i}$ for $q \geq 1$, which indicates that a greater $\lambda_{i}$ will result in reduction 
of the amount of pixels classified as the $i$-th tissue. To demonstrate the impact of $\lambda_{i}$ visually, we segment the 128-th image slice of IBSR_09 with nine different values of $\lambda_{2}$ equally spaced in the interval of [0.4,2.0] where $\lambda_{1}$ and $\lambda_{3}$ are both set to be 2.0 and show the results in Fig. 23. In this experiment, cluster centroids $\left\{c_{i}\right\}_{i=1}^{3}$ are initialized as equally spaced values in the range of intensities for the image. It can be seen from the original image as shown in Fig. 23(a), the intensities of GM are lower than WM but greater than CSF. Therefore, the increase of $\lambda_{2}$ will decrease the amount of pixels classified as GM as shown in Fig. 23(b), which can be also seen visually from GM segmented by BCEFCM with $\lambda_{2}$ changing with step 0.2 in the interval of $[0.4,2.0]$ as shown in Fig. 23) (c) to Fig. 23(k).

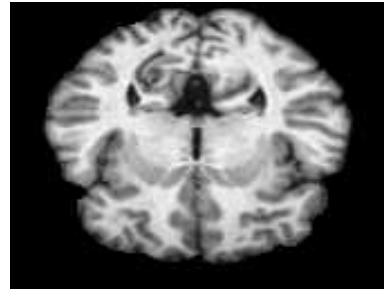

(a) The original image

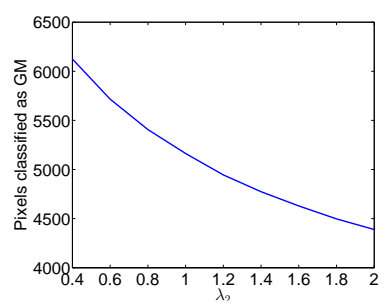

(b) The amount curve of GM

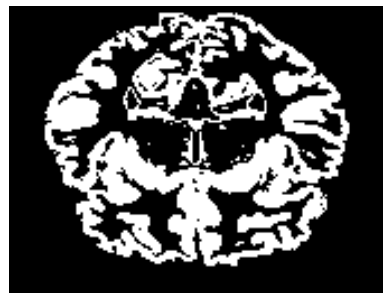

(c) $\lambda_{2}=0.4$

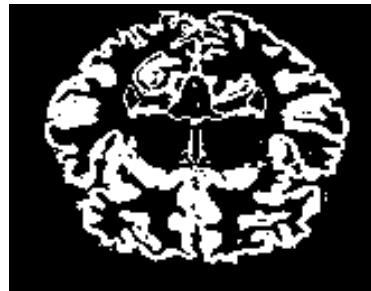

(f) $\lambda_{2}=1.0$

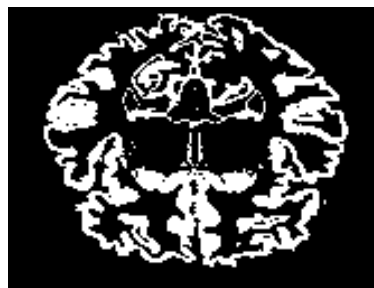

(i) $\lambda_{2}=1.6$

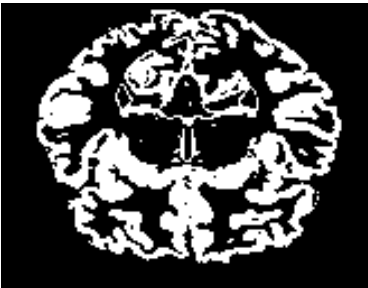

(d) $\lambda_{2}=0.6$

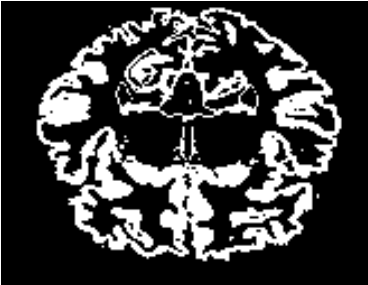

(g) $\lambda_{2}=1.2$

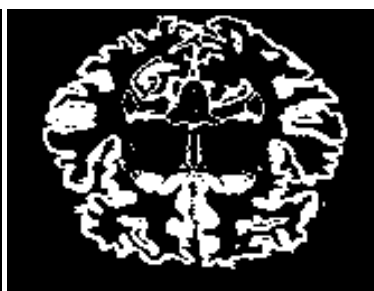

(j) $\lambda_{2}=1.8$

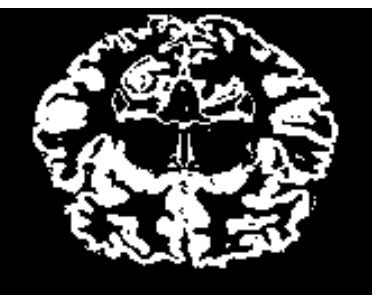

(e) $\lambda_{2}=0.8$

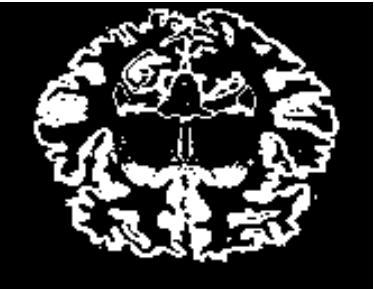

(h) $\lambda_{2}=1.4$

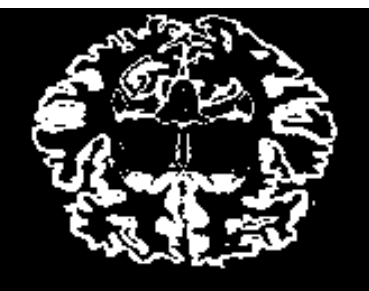

(k) $\lambda_{2}=2.0$

Figure 23: Demonstration of the impact of parameter $\lambda_{2}$ on the size of GM segmented by BCEFCM when $\lambda_{1}$ and $\lambda_{3}$ are both set to be 2.0 . 


\subsection{Impact of Parameter $\rho$}

As mentioned earlier, the bias field is smoothed once it is updated in each iteration by convoluting it with a kernel and the averaging filter $K_{\rho}$ is used in this paper. Note that the size (element number) of convolution kernel $K_{\rho}$ is controlled by the parameter $\rho$. In fact, the greater the parameter $\rho$ is, the larger the scale of $K_{\rho}$ is and the smoother the estimated bias field is. To demonstrate the impact of $\rho$ on the smoothness of the estimated bias field and on the quality of the corrected image visually, we segment the 90-th image slice of BrainWeb which is corrupted by slight noise and severe intensity inhomogeneity with $\rho$ taking odd values in $[1,11]$ and give the results in Fig. 24. We can see that the estimated bias fields are smooth enough and the bias corrected images are good enough if the parameter $\rho$ of BCEFCM takes values that are not less than 7 .

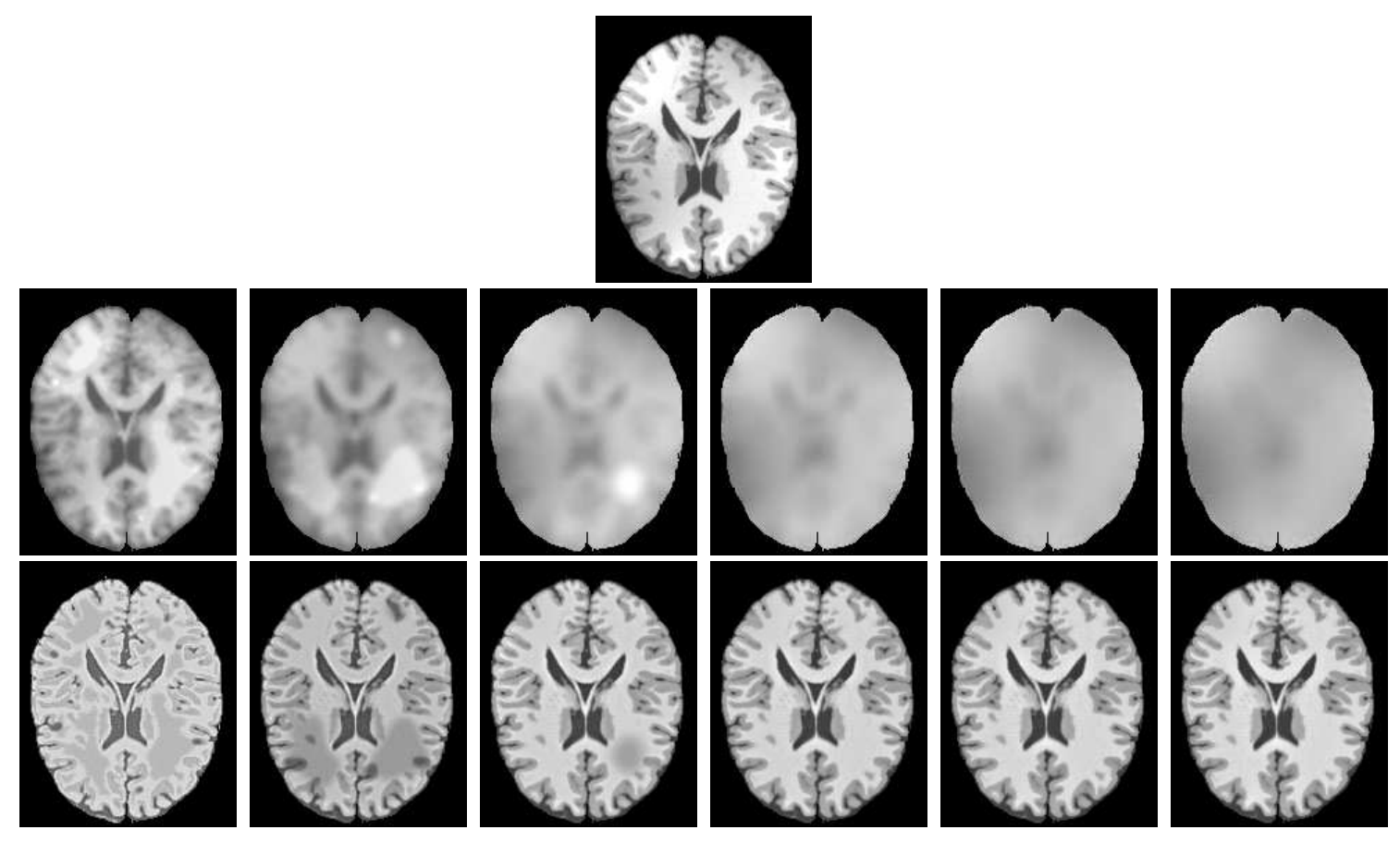

Figure 24: Demonstration of the impact of parameter $\rho$ on the smoothness of the estimated field and on the quality of the corrected image. The original image is given in the 1st row. The estimated bias fields and corrected images obtained from BCEFCM with parameter $\rho=1,3,5,7,9,11$ are given in the 2nd and 3rd rows from left to right, respectively.

\section{Conclusion}

A image segmentation framework is proposed in this paper to segment images with both additive noise and multiplicative intensity inhomogeneity. In the framework, a CUDA accelerated non-local means denosing method is first used to remove the additive noise. Then, a bias correction embedded FCM method is proposed to segment the image with multiplicative intensity inhomogeneity. The proposed framework has been extensively tested on both selected synthetic and real images and public BSD, BrainWeb and IBSR 
datasets. Experimental results and comparison analysis demonstrate that the proposed framework 1) is not sensitive to the intialization, 2) is able to dealt with noise and correct the bias field, and 3) is also faster and more accurate than state-of-the-art methods. In the near future, we will implement the remaining of the proposed method on GPU using CUDA to further improve its time performance. For images from BSD, We found that numbers of object in each of the image are quite different and the ground truths tend to recognize primary objects in the image. Unfortunately, we need to know in advance how many classes the image should be segmented into and our method is pixel-based which will recognize objects even they are small. Therefore, we will also improve the proposed method in the future to roughly estimate numbers of object in the image in advance by analysing statistical information of its intensities and will introduce a multiple-phase level set method to further refine segmentation results of the proposed method.

\section{Acknowledgements}

This work was supported by the Fundamental Research Funds for the Central Universities of China under grant N140403006 and N140407001, the National Science Foundation for Distinguished Young Scholars of China under Grant No. 71325002, No. 61225012, the National Science Foundation of China under grant No. 61172002 and No.71071028, the Postdoctoral Scientific Research Funds of Northeastern University under grant No. 20150310, the National Key Technology Research and Development Program of the Ministry of Science and Technology of China under grant 2014BAI17B01, and the Fundamental Research Funds for State Key Laboratory of Synthetical Automation for Process Industries under Grant No.2013ZCX11. The authors would like to thank the editor for holding and considering to publish our paper and would also like to greatly appreciate the anonymous reviewers for their valuable comments to improve the quality of this paper.

\section{References}

[1] Y. Wu, C. He, A convex variational level set model for image segmentation, Signal Processing 106 (2015) $123-133$.

[2] D. E. Ilea, P. F. Whelan, Image segmentation based on the integration of colour-texture descriptors-A review, Pattern Recognition 44 (10) (2011) 2479-2501.

[3] N. R. Pal, S. K. Pal, A review on image segmentation techniques, Pattern Recognition 26 (9) (1993) $1277-1294$.

[4] D. L. Pham, C. Xu, J. L. Prince, Current methods in medical image segmentation 1, Annual review of biomedical engineering 2 (1) (2000) 315-337.

[5] T. Liu, D. Tao, On the performance of Manhattan nonnegative matrix factorization, IEEE Transactions on Neural Networks and Learning Systems pp (99) (2015) 1-13.

[6] N. Guan, D. Tao, Z. Luo, J. Shawe-Taylor, MahNMF: Manhattan non-negative matrix factorization, arXiv preprint arXiv:1207.3438.

[7] N. Guan, D. Tao, Z. Luo, B. Yuan, NeNMF: an optimal gradient method for nonnegative matrix factorization, IEEE Transactions on Signal Processing 60 (6) (2012) 2882-2898. 
[8] M. A. Balafar, A. R. Ramli, M. I. Saripan, S. Mashohor, Review of brain MRI image segmentation methods, Artificial Intelligence Review 33 (3) (2010) 261-274.

[9] Z. Ma, J. M. R. Tavares, R. N. Jorge, T. Mascarenhas, A review of algorithms for medical image segmentation and their applications to the female pelvic cavity, Computer Methods in Biomechanics and Biomedical Engineering 13 (2) (2010) $235-246$.

[10] Y. Ou, J. Doshi, G. Erus, C. Davatzikos, Multi-atlas segmentation of the prostate: A zooming process with robust registration and atlas selection, Medical Image Computing and Computer Assisted Intervention (MICCAI) Grand Challenge: Prostate MR Image Segmentation 7 (2012) 1-7.

[11] Y. Ou, D. Shen, M. Feldman, J. Tomaszewski, C. Davatzikos, Non-rigid registration between histological and MR images of the prostate: A joint segmentation and registration framework, in: Computer Vision and Pattern Recognition Workshops, 2009. CVPR Workshops 2009. IEEE Computer Society Conference on, IEEE, 2009, pp. 125-132.

[12] W. Liu, D. Tao, J. Cheng, Y. Tang, Multiview hessian discriminative sparse coding for image annotation, Computer Vision and Image Understanding 118 (2014) 50-60.

[13] C. Hong, J. Yu, J. Wan, D. Tao, M. Wang, Multimodal deep autoencoder for human pose recovery, IEEE Transactions on Image Processing 24 (12) (2015) 5659-5670.

[14] C. Xu, D. Tao, C. Xu, Multi-view intact space learning, IEEE Transactions on Pattern Analysis and Machine Intelligence 37 (12) (2015) 2531-2544.

[15] C. Xu, D. Tao, C. Xu, Multi-view learning with incomplete views, IEEE Transactions on Image Processing 24 (12) (2015) $5812-5825$.

[16] W. Liu, D. Tao, Multiview hessian regularization for image annotation, IEEE Transactions on Image Processing 22 (7) (2013) 2676-2687.

[17] B. Wang, X. Gao, D. Tao, X. Li, A unified tensor level set for image segmentation, IEEE Transactions on Systems, Man, and Cybernetics, Part B: Cybernetics 40 (3) (2010) 857-867.

[18] B. Wang, X. Gao, D. Tao, X. Li, A nonlinear adaptive level set for image segmentation, IEEE Transactions on Cybernetics 44 (3) (2014) 418-428.

[19] C. Feng, C. Li, D. Zhao, C. Davatzikos, H. Litt, Segmentation of the left ventricle using distance regularized two-layer level set approach, in: Medical Image Computing and Computer-Assisted Intervention-MICCAI 2013, Springer, 2013, pp. $477-484$

[20] M. Filippone, F. Camastra, F. Masulli, S. Rovetta, A survey of kernel and spectral methods for clustering, Pattern Recognition 41 (1) (2008) 176-190.

[21] J. Nayak, B. Naik, H. Behera, Fuzzy c-means (FCM) clustering algorithm: A decade review from 2000 to 2014, in: Computational Intelligence in Data Mining-Volume 2, Springer, 2015, pp. 133-149.

[22] U. Vovk, F. Pernus, B. Likar, A review of methods for correction of intensity inhomogeneity in MRI, IEEE Transactions on Medical Imaging 26 (3) (2007) 405-421.

[23] R. Suganya, R. Shanthi, Fuzzy c-means algorithm-a review, International Journal of Scientific and Research Publications 2 (11) (2012) 1 .

[24] F. Zhao, L. Jiao, H. Liu, X. Gao, A novel fuzzy clustering algorithm with non local adaptive spatial constraint for image segmentation, Signal Processing 91 (4) (2011) 988-999.

[25] X.-Y. Wang, J. Bu, A fast and robust image segmentation using FCM with spatial information, Digital Signal Processing 20 (4) (2010) 1173-1182.

[26] J. Feng, L. Jiao, X. Zhang, M. Gong, T. Sun, Robust non-local fuzzy c-means algorithm with edge preservation for SAR image segmentation, Signal Processing 93 (2) (2013) 487-499.

[27] L. Liao, T. Lin, B. Li, MRI brain image segmentation and bias field correction based on fast spatially constrained kernel 
clustering approach, Pattern Recognition Letters 29 (10) (2008) 1580-1588.

[28] M. Ahmed, S. Yamany, A. Farag, T. Moriarty, A modified fuzzy c-means algorithms for bias field estimation and segmentation of MRI data, IEEE Transactions on Medical Imaging 21 (3) (2002) 193-199.

[29] S. Chen, D. Zhang, Robust image segmentation using FCM with spatial constraints based on new kernel-induced distance measure, IEEE Transactions on Systems, Man, and Cybernetics, Part B: Cybernetics 34 (4) (2004) $1907-1916$.

[30] M.-S. Yang, H.-S. Tsai, A gaussian kernel-based fuzzy c-means algorithm with a spatial bias correction, Pattern Recognition Letters 29 (12) (2008) 1713-1725.

[31] C. Li, J. C. Gore, C. Davatzikos, Multiplicative intrinsic component optimization (MICO) for MRI bias field estimation and tissue segmentation, Magnetic Resonance Imaging 32 (7) (2014) 913-923.

[32] A. A.-T. Ahmed Al-Taie, H. K. H. Horst K. Hahn, L. L. Lars Linsen, Improved bias-corrected fuzzy c-means segmentation of brain mri data, International Journal of Hybrid Information Technology 7 (3) (2014) 65-84.

[33] A. Buades, B. Coll, J.-M. Morel, Nonlocal image and movie denoising, International Journal of Computer Vision 76 (2) (2008) 123-139.

[34] J. V. Manjón, J. Carbonell-Caballero, J. J. Lull, G. García-Martí, L. Martí-Bonmatí, M. Robles, Mri denoising using non-local means, Medical Image Analysis 12 (4) (2008) 514-523.

[35] A. Buades, B. Coll, J.-M. Morel, A review of image denoising algorithms, with a new one, Multiscale Modeling \& Simulation 4 (2) (2005) 490-530.

[36] J. V. Manjón, P. Coupé, A. Buades, MRI noise estimation and denoising using non-local PCA, Medical image analysis 22 (1) (2015) 35-47.

[37] P. Coupé, P. Yger, C. Barillot, Fast non local means denoising for 3D MR images, in: Medical Image Computing and Computer-Assisted Intervention-MICCAI 2006, Springer, 2006, pp. 33-40.

[38] A. Eklund, P. Dufort, D. Forsberg, S. M. LaConte, Medical image processing on the GPU-Past, present and future, Medical Image Analysis 17 (8) (2013) 1073-1094.

[39] J. Yang, C. Feng, D. Zhao, A CUDA-based reverse gridding algorithm for MR reconstruction, Magnetic Resonance Imaging 31 (2) (2013) 313-323.

[40] C. Feng, J. Yang, D. Zhao, J. Liu, CUDA accelerated method for motion correction in MR PROPELLER imaging, Magnetic Resonance Imaging 31 (8) (2013) 1390-1398.

[41] C. Feng, D. Zhao, CUDA accelerated uniform re-sampling for non-Cartesian MR reconstruction, Bio-Medical Materials and Engineering 26 (s1) (2015) 983-989.

[42] D. Martin, C. Fowlkes, D. Tal, J. Malik, A database of human segmented natural images and its application to evaluating segmentation algorithms and measuring ecological statistics, in: Proc. 8th Int'l Conf. Computer Vision, Vol. 2, 2001, pp. 416-423.

[43] C. A. Cocosco, V. Kollokian, R. K.-S. Kwan, G. B. Pike, A. C. Evans, Brainweb: online interface to a 3D MRI simulated brain database, in: NeuroImage, Citeseer, 1997.

[44] T. Rohlfing, Image similarity and tissue overlaps as surrogates for image registration accuracy: widely used but unreliable, IEEE Transactions on Medical Imaging 31 (2) (2012) 153-163. 